Pacific

Journal of

Mathematics

ASCENT AND DESCENT FOR FINITE SEQUENCES OF COMMUTING ENDOMORPHISMS

LuZius GRUnenfelder AND Matjaž OMLadič 


\title{
ASCENT AND DESCENT FOR FINITE SEQUENCES OF COMMUTING ENDOMORPHISMS
}

\author{
Luzius Grunenfelder and Matjaž Omladič
}

\begin{abstract}
Homological techniques involving the Koszul complex are used to define and explore two invariants, ascent and descent, for a finite sequence of commuting endomorphism of a module. It is shown in particular that, as in the case of a single endomorphism, if ascent and descent are both finite then they are equal, and that this finiteness condition is equivalent to a certain strong Fitting type property.
\end{abstract}

\section{Introduction.}

Let $\mathcal{A}$ be an algebra over a commutative $\operatorname{ring} R$ and let $\mathcal{M}$ be a left $\mathcal{A}$ module. If $a: \mathcal{M} \rightarrow \mathcal{M}$ is an $\mathcal{A}$-endomorphism then ker $a^{i} \subseteq \operatorname{ker} a^{i+1}$ and $\operatorname{im} a^{i} \supseteq \operatorname{im} a^{i+1}$ for every $i \geq 0$. The ascent of $a$ is the least positive integer $r$ for which $\operatorname{ker} a^{r}=\operatorname{ker} a^{r+1}$ and the descent is the least positive integer $s$ for which $\operatorname{im} a^{s}=\operatorname{im} a^{s+1}$, if such integers exist and $\infty$ if they don't. If both the ascent $r$ and the descent $s$ of $a$ are finite then $r=s$ and $\mathcal{M}=\operatorname{ker} a^{r} \oplus \operatorname{im} a^{r}$. This is Fitting's Lemma. It holds in particular for every $a \in \operatorname{End}_{\mathcal{A}}(\mathcal{M})$ if the $\mathcal{A}$-module $\mathcal{M}$ is both Artinian and Noetherian. More generally, we may say that $a$ has the Fitting property if $\mathcal{M}=\mathcal{K} \oplus \mathcal{I}$, where $\mathcal{K}=\cup_{r}$ ker $a^{r}$ and $\mathcal{I}=\cap_{r} a^{r} \mathcal{M}$.

In this paper we consider $n$-tuples $\mathbf{a}=\left(a_{1}, a_{2}, \ldots, a_{n}\right)$ of commuting endomorphisms of the $\mathcal{A}$-module $\mathcal{M}$. The ascent and the descent of a are defined and investigated by means of homological techniques. The role of the single endomorphism $a$ in the classical case will be taken by the Koszul complex $K\left(\mathcal{M}, \partial_{\mathbf{a}}\right)$ of the $n$-tuple $\mathbf{a}$. Composition by the endomorphism $a$ is replaced by the procedure of forming the diagonal complex of the double complex obtained via tensoring by the Koszul complex $K\left(A, \partial_{\mathbf{a}}\right)$, where $A$ is a commutative subalgebra of $\operatorname{End}_{\mathcal{A}}(\mathcal{M})$ containing the $n$-tuple a. This leads to a natural extension of the concepts of ascent, descent and of Fitting's Lemma to finite sequences of commuting endomorphisms in Sections 2 to 5 .

The whole approach works for any module $\mathcal{M}$ over a commutative ring $A$ and any finite sequence of elements of $A$, without specific reference to $R$ and to an $\mathcal{A}$-module structure on $\mathcal{M}$. However, in our context the Fitting decomposition is of course $\mathcal{A}$-invariant. In general, we say that the $n$-tuple a has the Fitting property if $\mathcal{M}=\mathcal{K} \oplus \mathcal{I}$ and $\mathfrak{a} \mathcal{I}=\mathcal{I}$, where 
$\mathcal{K}=\cup_{r} \operatorname{Hom}_{A}\left(A / \mathfrak{a}^{r}, \mathcal{M}\right), \mathcal{I}=\cap_{s} \mathfrak{a}^{s} \mathcal{M}$ and $\mathfrak{a}$ is the ideal in $A$ generated by the $n$-tuple a. The main result of Section 5 is that a has finite ascent and finite descent if and only if a has the Fitting property and $\mathfrak{a}$ acts nilpotently on $\mathcal{K}$.

In case $R$ is a field, we show in Section 7 and 8 that the concepts of ascent and descent are actually functions defined on finite dimensional subspaces of commuting elements of $\operatorname{End}_{\mathcal{A}}(\mathcal{M})$. More precisely, if the commuting $n$ tuple $\mathbf{a}$ and the commuting $m$-tuple $\mathbf{b}$ span the same subspace in $\operatorname{End}_{\mathcal{A}}(\mathcal{M})$ then they yield the same ascent, the same descent and the same Fitting decomposition of $\mathcal{M}$. Localization techniques are then employed to see that these concepts are functions defined on finitely generated commutative subalgebras of $\operatorname{End}_{\mathcal{A}}(\mathcal{M})$, i.e. if the finite sequences $\mathbf{a}$ and $\mathbf{b}$ generate the same ideal in the commutative subalgebra $A$ of $\operatorname{End}_{\mathcal{A}}(\mathcal{M})$ then they yield the same ascent and the same descent, even in the absence of the Fitting property.

Apart from their independent interest, the results presented in this paper have also been motivated by some open problems in functional analysis and operator theory. It is folk knowledge in this theory that the spectral behaviour of a single compact operator in the vicinity of a non-zero spectral point can be studied using an analog of Fitting's Lemma [TAE, p. 271ff]. It was J.S. Taylor $[\mathbf{T}]$ who introduced the Koszul complex into the discussion of the joint spectrum of a commuting $n$-tuple of bounded operators. The question of how to study the spectral behaviour of such an $n$-tuple is of considerable importance, in particular in applications like multiparameter spectral theory $[\mathbf{R}]$ and the theory of elementary operators $[\mathbf{C}]$. In $[\mathbf{B T}]$ the Taylor spectrum was applied to study the spectral properties of a commuting $n$-tuple of compact operators by combining the ideas of Fitting's lemma and the Koszul complex. Our approach provides a Fitting type decomposition for any point in the Taylor spectrum. To establish notation and for the convenience of the reader some of the well-known results concerning the homology of Koszul complexes [S] are reviewed in Section 2.

\section{Preliminaries on Koszul Complexes.}

2.1. Throughout this article let $\mathcal{A}$ be a fixed associative algebra with unit over the commutative ring $R$, and let $\mathcal{M}$ be a fixed left $\mathcal{A}$-module. Furthermore, let $A$ be a commutative subalgebra of $\operatorname{End}_{\mathcal{A}}(\mathcal{M})$. For any $n$-tuple $\mathbf{a}=\left(a_{1}, a_{2}, \ldots, a_{n}\right)$ of elements of $A$ we may construct the Koszul complexes $K(A, \mathbf{a})$ and $K(\mathcal{M}, \mathbf{a})$ as follows. If $\mathbf{e}=\left(e_{1}, e_{2}, \ldots, e_{n}\right)$ is a basis of the free $A$-module $A^{n}$ then the differential graded $A$-algebra $K(A, \mathbf{a})$ consists of the exterior algebra $\Lambda\left(A^{n}\right)$ together with the differential of degree one

$$
\partial_{\mathbf{a}}: \Lambda\left(A^{n}\right) \rightarrow \Lambda\left(A^{n}\right)
$$


defined by $\partial_{\mathbf{a}}(x)=\sum_{i=1}^{n} a_{i} e_{i} \wedge x$. The Koszul cochain complex of $\mathcal{M}$ is then the differential graded $A$-module $K(\mathcal{M}, \mathbf{a})=\mathcal{M} \otimes_{A} K(A, \mathbf{a})$. It is often useful to use the recursive definition $K(A, \mathbf{a})=K\left(A, \mathbf{a}^{\prime}\right) \otimes_{A} K\left(A, a_{n}\right)$ of the Koszul complex, where $\mathbf{a}^{\prime}=\left(a_{1}, a_{2}, \ldots, a_{n-1}\right)$ and where $K\left(A, a_{n}\right)$ is just the complex $a_{n}: A \rightarrow A$.

Proposition 2.2. Let $X$ be any cochain complex of $A$-modules and let a be an element of $A$. Then there is a short exact sequence in cohomology

$$
\begin{aligned}
0 \rightarrow H^{1}\left(H^{p-1}(X) \otimes_{A} K(A, a)\right) \rightarrow H^{p}\left(X \otimes_{A} K(A, a)\right) & \\
& \rightarrow H^{0}\left(H^{p}(X) \otimes_{A} K(A, a)\right) \rightarrow 0
\end{aligned}
$$

for each integer $p \geq 0$.

Proof. Considering $K(A, a)$ as the complex $a: K^{0}(A, a) \rightarrow K^{1}(A, a)$ then we get the short exact sequence of cochain complexes

$$
0 \rightarrow\left(X \otimes_{A} K^{1}(A, a)\right)^{p-1} \rightarrow\left(X \otimes_{A} K(A, a)\right)^{p} \rightarrow\left(X \otimes_{A} K^{0}(A, a)\right)^{p} \rightarrow 0
$$

and the associated long exact sequence in cohomology

$$
\begin{aligned}
H^{p-1}(X) \otimes_{A} K^{0}(A, a) & \stackrel{1 \otimes_{A} a}{\longrightarrow} H^{p-1}(X) \otimes_{A} K^{1}(A, a) \rightarrow H^{p}\left(X \otimes_{A} K(A, a)\right) \\
& \rightarrow H^{p}(X) \otimes_{A} K^{0}(A, a) \stackrel{1 \otimes_{A} a}{\longrightarrow} H^{p}(X) \otimes_{A} K^{1}(A, a) .
\end{aligned}
$$

The assertion is now established, by taking the kernel of $1 \otimes_{A} a$ on the right $H^{0}\left(H^{p}(X) \otimes_{A} K(A, a)\right)=\operatorname{ker}\left(1 \otimes_{A} a\right)=\operatorname{ker}\left(H^{p-1}(X) \stackrel{a}{\rightarrow} H^{p-1}(X)\right)$ and of course its cokernel on the left $H^{1}\left(H^{p-1}(X) \otimes_{A} K(A, a)\right)=\operatorname{coker}\left(1 \otimes_{A} a\right)=$ $H^{p-1}(X) / a H^{p-1}(X)$.

Corollary 2.3. Let $X$ be any cochain complex of $A$-modules and let a be an element of $A$. If $a$ is invertible then $H^{*}\left(X \otimes_{A} K(A, a)\right)=0$ and if $a=0$ then $H^{p}\left(X \otimes_{A} K(A, a)\right) \cong H^{p-1}(X) \oplus H^{p}(X)$.

Corollary 2.4. If $\mathcal{M} \neq 0$ is Artinian and Noetherian as an A-module and if $\mathbf{a} \subset \operatorname{rad} A$ then the $H^{p}(K(\mathcal{M}, \mathbf{a})) \neq 0$ for $0 \leq p \leq n$.

Proof. Let $\mathbf{a}=\left(a_{1}, a_{2}, \ldots, a_{n}\right)$ be contained in the Jacobson radical $\operatorname{rad} A$. The result will be established by induction on $n$. For $n=1$ the exact sequence

$$
0 \rightarrow H^{0}(\mathcal{M}, a) \rightarrow \mathcal{M} \stackrel{a}{\rightarrow} \mathcal{M} \rightarrow H^{1}(\mathcal{M}, a) \rightarrow 0
$$

of Artinian and Noetherian $A$-modules describes the situation. If $H^{0}(\mathcal{M}, a)$ $=0$ then the map $a: \mathcal{M} \rightarrow \mathcal{M}$ is injective. The descending chain of submodules

$$
\mathcal{M} \supseteq \operatorname{im} a \supseteq \operatorname{im} a^{2} \supseteq \ldots \supseteq \operatorname{im} a^{j} \supseteq \ldots
$$

must become stationary after finitely many steps since $\mathcal{M}$ is Artinian. But, $\operatorname{im} a^{j}=\operatorname{im} a^{j+1}=a\left(\operatorname{im} a^{j}\right)$ means that $a^{j}(\operatorname{im} a)=a^{j}(\mathcal{M})$ so that $a \mathcal{M}=$ $\operatorname{im} a=\mathcal{M}$, since $a^{j}$ is injective. But if $H^{1}(\mathcal{M}, a)=\mathcal{M} / a \mathcal{M}=0$ then 
$\mathcal{M}=0$ by Nakayama's Lemma, hence a contradiction. Thus, the case $n=1$ is established. Now assume that $n>1, \mathbf{a}^{\prime}=\left(a_{1}, a_{2}, \ldots, a_{n-1}\right)$. The induction hypothesis is that the $A$-modules $H^{q}\left(\mathcal{M}, \mathbf{a}^{\prime}\right) \neq 0$ for all $q$ satisfying $0 \leq q \leq n-1$ and that they are both Artinian and Noetherian. If $H^{p}(\mathcal{M}, \mathbf{a})=0$ then by the exact sequences of Proposition 2.2

$$
\begin{aligned}
0 \rightarrow H^{1}\left(H^{p-1}(X) \otimes_{A} K(A, a)\right) \rightarrow H^{p}\left(X \otimes_{A} K(A, a)\right) & \\
& \rightarrow H^{0}\left(H^{p}(X) \otimes_{A} K(A, a)\right) \rightarrow 0
\end{aligned}
$$

with $X=K\left(\mathcal{M}, \mathbf{a}^{\prime}\right)$ and $a=a_{n}$, we see that $H^{p-1}(X) / a_{n} H^{p-1}(X)=0$ and $\operatorname{ker}\left(a_{n}: H^{p}(X) \rightarrow H^{p}(X)\right)=0$. For $p>0$, Nakayama's Lemma implies that $H^{p-1}\left(\mathcal{M}, \mathbf{a}^{\prime}\right)=0$, which contradicts the induction hypothesis unless $p>n$. If $p=0$, then $H^{0}(\mathcal{M}, \mathbf{a})=\operatorname{ker}\left(a_{n}: H^{0}\left(\mathcal{M}, \mathbf{a}^{\prime}\right) \rightarrow H^{0}\left(\mathcal{M}, \mathbf{a}^{\prime}\right)\right)=0$ so that $a_{n}: H^{0}\left(\mathcal{M}, \mathbf{a}^{\prime}\right) \rightarrow H^{0}\left(\mathcal{M}, \mathbf{a}^{\prime}\right)$ is an injective endomorphism of the Artinian $A$-module $H^{0}\left(\mathcal{M}, \mathbf{a}^{\prime}\right)$, hence bijective. Again Nakayama's Lemma implies that $H^{0}\left(\mathcal{M}, \mathbf{a}^{\prime}\right)=0$ in contradiction to the induction hypothesis.

Corollary 2.5. For any $\mathcal{A}$-module $\mathcal{M}$ the commuting $n$-tuple $\mathbf{a}$ is in the annihilator of $H^{*}(K(\mathcal{M}, \mathbf{a}))$.

Proof. This follows easily by induction on $n$ using the exact sequence of Proposition 2.2 with $X=K\left(\mathcal{M}, \mathbf{a}^{\prime}\right)$ and $a=a_{n}$.

2.6. If $X$ is a cochain complex of $A$-modules then $X \otimes_{A} K(A$,a) becomes a bicomplex with differentials $\partial \otimes_{A} 1$ and $1 \otimes_{A} \partial_{\mathbf{a}}$. The diagonal complex $D=D\left(X \otimes_{A} K(A, \mathbf{a})\right)$ with $D_{i}=X_{i} \otimes_{A} K_{i}$ has differential $\partial \otimes_{A} \partial_{\mathbf{a}}$. Let $K^{(0)}=A=D^{(0)}$ and for $r \geq 1$ define inductively the iterated diagonal complex $D^{(r)}=D\left(D^{(r-1)} \otimes_{A} K(A, \mathbf{a})\right)$, which is a differential graded $A$ algebra with $D_{i}^{(r)}=\otimes_{A}^{r} K_{i}(A, \mathbf{a})$ and differential $\partial^{(r)}=\otimes_{A}^{r} \partial_{\mathbf{a}}=\partial^{1} \partial^{2} \ldots \partial^{r}$, where $\partial^{j}: \otimes_{A}^{r} K_{i}(A, \mathbf{a}) \rightarrow \otimes_{A}^{r} K_{i+1}(A, \mathbf{a})$ is acting like $\partial_{\mathbf{a}}$ on the $j$-th and like the identity on the remaining tensor factors. Finally we introduce the differential graded $D^{(r)} A$-module $\mathcal{M}^{(r)}=\mathcal{M} \otimes_{A} D^{(r)}$. Since there is no danger of confusion, we shall use the same notation $\partial^{j}$ and $\partial^{(r)}$ for the corresponding differentials on both $D^{(r)}$ and $\mathcal{M}^{(r)}$.

From the degree-wise and coordinate-wise point of view we may introduce an additional upper index on the basis elements indicating the factor in the tensor power $D_{i}^{(r)}=\otimes_{A}^{r} K_{i}(A, \mathbf{a})$. A basis of $D_{p}^{(r)}$ is given by $\left\{e_{I_{1}}^{1} \otimes_{A} e_{I_{2}}^{2} \otimes_{A}\right.$ $\left.\ldots \otimes_{A} e_{I_{r}}^{r}\right\}$, where $e_{I}=e_{i_{1}} \wedge e_{i_{2}} \wedge \ldots \wedge e_{i_{p}}$ for the multi-index $I=\left(i_{1}, i_{2}, \ldots, i_{p}\right)$ of type $p$ with $1 \leq i_{1}<i_{2}<\cdots<i_{p} \leq n$. Notice that $\mathcal{M}_{i}^{(r)}=\mathcal{M} \otimes_{A} D_{i}^{(r)}$ and that $\partial_{i}^{(r)}: \mathcal{M}_{i}^{(r)} \rightarrow \mathcal{M}_{i+1}^{(r)}$ is the composite $\partial_{i}^{(r)}=\partial_{i}^{1} \partial_{i}^{2} \ldots \partial_{i}^{r}$, where $\partial_{i}^{j} x=\left(\sum_{k=1}^{n} a_{k} e_{k}^{j}\right) \wedge x$ for every $x \in \mathcal{M}_{i}^{(r)}$. We can also define $\mathcal{M}_{i}^{(r)}$ and the differentials $\partial_{i}^{(r)}$ inductively using the commutative diagram in Figure 1. Observe that the main diagonal of this commutative diagram represents the 
left most vertical of the diagram on the next step, i.e. with $r$ replaced by $r+$ 1. Every row and every column of the diagram represents a cochain complex. Also, the left most and the right most columns are actually isomorphic. Since $D_{j}$ is a free $A$-module, the cohomology of the $j$-th column is

$$
H^{*}\left(\mathcal{M}^{(r)} \otimes_{A} D_{j}\right) \cong H^{*}\left(\mathcal{M}^{(r)}\right) \otimes_{A} D_{j} .
$$

The $i$-th row is of course isomorphic to the Koszul complex

$$
K\left(\mathcal{M}_{i}^{(r)}, \mathbf{a}\right) \cong K(\mathcal{M}, \mathbf{a}) \otimes_{A} D_{i}^{(r)}
$$

of the $A$-module $M_{i}^{(r)} \cong \mathcal{M} \otimes_{A} D_{i}^{(r)}$ and, since $D_{i}^{(r)}$ is a free $A$-module, its cohomology is

$$
H^{*}\left(K\left(\mathcal{M}_{i}^{(r)} \otimes_{A} D, \mathbf{a}\right)\right) \cong H^{*}(K(\mathcal{M}, \mathbf{a})) \otimes_{A} D_{i}^{(r)} .
$$

In particular, if the $A$-module $\mathcal{M}$ is both Artinian and Noetherian and if $\mathbf{a} \subset \operatorname{rad} A$, then it follows from Corollary 2.4 that $H^{p}\left(K\left(\mathcal{M}_{i}^{(r)} \otimes_{A} D\right)\right) \neq 0$ for $0 \leq p \leq n$.

$$
\begin{aligned}
& \begin{array}{cccccc}
\mathcal{M}_{0}^{(r)} \stackrel{\partial_{0}^{r+1}}{\longrightarrow} \mathcal{M}_{0}^{(r)} \otimes_{A} D_{1} & \ldots & \mathcal{M}_{0}^{(r)} \otimes_{A} D_{n-1} & \stackrel{\partial_{n-1}^{r+1}}{\longrightarrow} & \mathcal{M}_{0}^{(r)} \otimes_{A} D_{n} \\
\downarrow \partial_{0}^{(r)} & \downarrow \partial_{0}^{(r)} & & \downarrow_{0}^{(r)} & & \\
& & & \partial_{0}^{(r)}
\end{array} \\
& \mathcal{M}_{1}^{(r)} \stackrel{\partial_{0}^{r+1}}{\longrightarrow} \mathcal{M}_{1}^{(r)} \otimes_{A} D_{1} \quad \ldots \quad \mathcal{M}_{1}^{(r)} \otimes_{A} D_{n-1} \stackrel{\partial_{n-1}^{r+1}}{\longrightarrow} \mathcal{M}_{1}^{(r)} \otimes_{A} D_{n} \\
& \downarrow \partial_{1}^{(r)} \quad \downarrow \partial_{1}^{(r)} \quad \downarrow \partial_{1}^{(r)} \quad \downarrow \partial_{1}^{(r)} \\
& \mathcal{M}_{2}^{(r)} \stackrel{\partial_{0}^{r+1}}{\longrightarrow} \mathcal{M}_{2}^{(r)} \otimes_{A} D_{1} \quad \ldots \quad \mathcal{M}_{2}^{(r)} \otimes_{A} D_{n-1} \stackrel{\partial_{n-1}^{r+1}}{\longrightarrow} \mathcal{M}_{2}^{(r)} \otimes_{A} D_{n}
\end{aligned}
$$

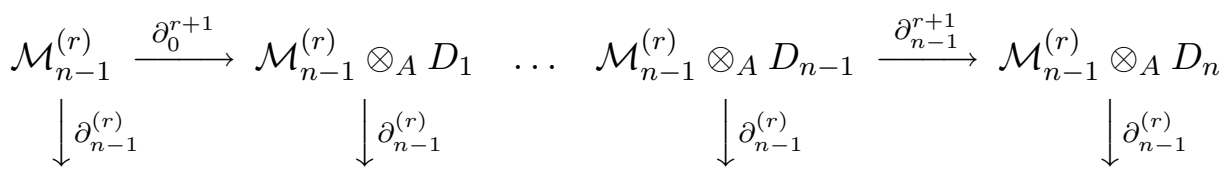

$$
\begin{aligned}
& \mathcal{M}_{n}^{(r)} \stackrel{\partial_{0}^{r+1}}{\longrightarrow} \mathcal{M}_{n}^{(r)} \otimes_{A} D_{1} \quad \ldots \quad \mathcal{M}_{n}^{(r)} \otimes_{A} D_{n-1} \stackrel{\partial_{n-1}^{r+1}}{\longrightarrow} \mathcal{M}_{n}^{(r)} \otimes_{A} D_{n}
\end{aligned}
$$

\section{Figure 1.}

The composite of any of either a horizontal or a vertical by a consecutive diagonal map is zero and so is the composite of any diagonal by a consecutive horizontal or vertical map. Therefore, any path which changes 
from horizontal to vertical direction or vice versa only through a diagonal is a cochain complex. Of particular interest are "Yoneda composites" of columns by rows and of rows by columns.

\section{Some Properties of the Double Complexes.}

At each point $\mathcal{M}_{i}^{(r)} \otimes_{A} D_{j}$ of the double complex in Fig. 1, i.e. at that point in the commutative diagram

$$
\begin{array}{ccc}
\mathcal{M}_{i-1}^{(r)} \otimes_{A} D_{j-1} & \stackrel{\partial_{j-1}^{r+1}}{\longrightarrow} \mathcal{M}_{i-1}^{(r)} \otimes_{A} D_{j} \stackrel{\partial_{j}^{r+1}}{\longrightarrow} \mathcal{M}_{i-1}^{(r)} \otimes_{A} D_{j+1} \\
\partial_{i-1}^{(r)} \downarrow & \partial_{i-1}^{(r)} \downarrow & \partial_{i-1}^{(r)} \downarrow \\
\mathcal{M}_{i}^{(r)} \otimes_{A} D_{j-1} \stackrel{\partial_{j-1}^{r+1}}{\longrightarrow} \mathcal{M}_{i}^{(r)} \otimes_{A} D_{j} \stackrel{\partial_{j}^{r+1}}{\longrightarrow} \mathcal{M}_{i}^{(r)} \otimes_{A} D_{j+1} \\
\partial_{i}^{(r)} \downarrow & \partial_{i}^{(r)} \downarrow & \partial_{i}^{(r)} \downarrow \\
\mathcal{M}_{i+1}^{(r)} \otimes_{A} D_{j-1} \stackrel{\partial_{j-1}^{r+1}}{\longrightarrow} \mathcal{M}_{i+1}^{(r)} \otimes_{A} D_{j} \stackrel{\partial_{j}^{r+1}}{\longrightarrow} \mathcal{M}_{i+1}^{(r)} \otimes_{A} D_{j+1}
\end{array}
$$

\section{Figure 2.}

we may consider the vertical, the vertical-diagonal and the diagonal-vertical cohomologies, denoted by $\mathcal{H}_{i j}^{(r)}, \mathcal{L}_{i j}^{(r)}$ and $\mathcal{R}_{i j}^{(r)}$, respectively. It follows in particular that

$$
H_{00}^{r}=\operatorname{ker} \partial_{0}^{(r)} \cong \operatorname{Hom}\left(A / \mathfrak{a}^{r}, \mathcal{M}\right) \quad, \quad H_{n n}^{(r)}=\mathcal{M} / \operatorname{im} \partial_{n-1}^{(r)} \cong M / \mathfrak{a}^{r} \mathcal{M},
$$

$\mathcal{L}_{00}^{(r)}=\mathcal{H}_{00}^{(r+1)}$ and $R_{n n}^{(r)}=H_{n n}^{(r+1)}$ for all $r \geq 1$, where $\mathfrak{a}$ is the ideal in $A$ generated by the $n$-tuple $\mathbf{a}$.

Proposition 3.1. Let $\mathbf{a}$ be an n-tuple of endomorphisms contained in the commutative subalgebra $A$ of $\operatorname{End}_{\mathcal{A}}(\mathcal{M})$.

(a) Suppose $0 \leq j<n$. If $\mathcal{L}_{l j}^{(r)}=\mathcal{H}_{l j}^{(r)}$ for some l, where $0 \leq l<n$, then $\mathcal{L}_{k j}^{(r)}=\mathcal{H}_{k j}^{(r)}$ for all $k$, where $0 \leq k \leq l$.

(b) Suppose $0<j \leq n$. If $\mathcal{R}_{l j}^{(r)}=\mathcal{H}_{l j}^{(r)}$ for some l, where $0<l \leq n$, then $\mathcal{R}_{k j}^{(r)}=\mathcal{H}_{k j}^{(r)}$ for all $k$, where $l \leq k \leq n$.

Proof. a) Observe that, due to the fact that the vertical and the verticaldiagonal cohomologies at a point are defined by the same incoming homomorphism, we only have to show that the outgoing maps have equal kernels. However, since the diagonal map $\partial_{j}^{r+1} \partial_{k}^{(r)}$ is a composite by the vertical map $\partial_{k}^{(r)}$, it suffices to prove that $\operatorname{ker}\left(\partial_{j}^{r+1} \partial_{k}^{(r)}\right) \subseteq \operatorname{ker}\left(\partial_{k}^{(r)}\right)$. In order to see this 
pick an element $x \in \mathcal{M}_{k}^{(r)} \otimes_{A} D_{j}$ from the kernel of the outgoing diagonal map and write

$$
x=\sum_{I_{1}, I_{2}, \ldots, I_{r+1}} v_{I_{1} I_{2} \ldots I_{r} I_{r+1}} e_{I_{1}} \otimes e_{I_{2}} \otimes \ldots \otimes e_{I_{r}} \otimes e_{I_{r+1}} .
$$

Here every $I_{i}$ for $1 \leq i \leq r$ is a multi-index of type $\left(j_{1}, j_{2}, \ldots, j_{k}\right)$, where $1 \leq j_{1}<j_{2}<\ldots<j_{k} \leq n$, and the corresponding $e_{I_{i}}=e_{j_{1}} \wedge e_{j_{2}} \wedge \ldots \wedge e_{j_{k}}$. Moreover, $I_{r+1}$ is a multi-index of type $\left(h_{1}, h_{2}, \ldots, h_{j}\right)$ with $1 \leq h_{1}<h_{2}<$ $\ldots<h_{j} \leq n$ and $e_{I_{r+1}}=e_{h_{1}} \wedge e_{h_{2}} \wedge \ldots \wedge e_{h_{j}}$. This implies that

$$
\begin{aligned}
\partial_{k}^{(r)} x= & \sum_{I_{1}, I_{2}, \ldots, I_{r+1}} \sum_{p_{1}, p_{2}, \ldots, p_{r}} a_{p_{1}} a_{p_{2}} \ldots a_{p_{r}} v_{I_{1} I_{2} \ldots I_{r} I_{r+1}} \\
& \left(e_{p_{1}} \wedge e_{I_{1}}\right) \otimes\left(e_{p_{2}} \wedge e_{I_{2}}\right) \otimes \ldots \otimes\left(e_{p_{r}} \wedge e_{I_{r}}\right) \otimes e_{I_{r+1}} .
\end{aligned}
$$

By assumption we have

$$
\begin{aligned}
& 0=\partial_{j}^{r+1} \partial_{k}^{(r)} x=\sum_{I_{1}, I_{2}, \ldots, I_{r+1}} \sum_{p_{1}, p_{2}, \ldots, p_{r+1}} a_{p_{1}} a_{p_{2}} \ldots a_{p_{r}} a_{p_{r+1}} v_{I_{1} I_{2} \ldots I_{r} I_{r+1}} \\
&\left(e_{p_{1}} \wedge e_{I 1}\right) \otimes\left(e_{p_{2}} \wedge e_{I_{2}}\right) \otimes \ldots \otimes\left(e_{p_{r}} \wedge e_{I_{r}}\right) \otimes\left(e_{p_{r+1}} \wedge e_{I_{r+1}}\right) .
\end{aligned}
$$

For $i=1,2, \ldots, r$ choose any multi-index $J_{i}$ of type $\left(j_{1}, j_{2}, \ldots, j_{l-k}\right)$, where $1 \leq j_{1}<j_{2}<\ldots<j_{l-k} \leq n$, and define $e_{J_{i}}=e_{j_{1}} \wedge e_{j_{2}} \wedge \ldots \wedge e_{j_{l-k}}$. Letting $e=e_{J_{1}} \otimes e_{J_{2}} \otimes \ldots \otimes e_{J_{r}}$, we see that $x \wedge e$ belongs to $\mathcal{M}_{l}^{r} \otimes_{A} D_{j}$. Now $\partial_{j}^{r+1} \partial_{l}^{(r)}(x \wedge e)=\left(\partial_{j}^{r+1} \partial_{l}^{(r)} x\right) \wedge e$, since the diagram

$$
\begin{array}{ccc}
\mathcal{M}_{k}^{(r)} \otimes_{A} D_{j} \stackrel{\partial_{k}^{(r)}}{\longrightarrow} \mathcal{M}_{k+1}^{(r)} \otimes_{A} D_{j} \stackrel{\partial_{j}^{r+1}}{\longrightarrow} \mathcal{M}_{k+1}^{(r)} \otimes_{A} D_{j+1} \\
\wedge e \downarrow & \wedge e \downarrow & \wedge e \downarrow \\
\mathcal{M}_{l}^{(r)} \otimes_{A} D_{j} \stackrel{\partial_{l}^{(r)}}{\longrightarrow} \mathcal{M}_{l+1}^{(r)} \otimes_{A} D_{j} \stackrel{\partial_{j}^{r+1}}{\longrightarrow} \mathcal{M}_{l+1}^{(r)} \otimes_{A} D_{j+1}
\end{array}
$$

is commutative. Thus, $x \in \operatorname{ker}\left(\partial_{j}^{r+1} \partial_{k}^{(r)}\right)$ implies that $x \wedge e \in \operatorname{ker}\left(\partial_{j}^{r+1} \partial_{l}^{(r)}\right)$ and by the assumptions in part a) also that $x \wedge e \in \operatorname{ker} \partial_{l}^{(r)}$ for every $e$ as described above. But then $\left(\partial_{k}^{(r)} x\right) \wedge e=\partial_{l}^{(r)}(x \wedge e)=0$ for every such $e$. Clearly there are sufficiently many maps $\wedge e$ of that kind to conclude that $\partial_{k}^{(r)} x=0$.

b) Similar arguments apply to the second part of the Proposition. First observe that at a given point both the vertical and the diagonal-vertical cohomologies are defined by the same outgoing homomorphism. It therefore suffices to prove that the ingoing maps have equal images. However, since the diagonal map $\partial_{k-1}^{(r)} \partial_{j-1}^{r}$ is a composite by the vertical map $\partial_{k-1}^{(r)}$, it remains to show that $\operatorname{im}\left(\partial_{k-1}^{(r)}\right) \subseteq \operatorname{im}\left(\partial_{k-1}^{(r)} \partial_{j-1}^{r}\right)$. To this effect pick $x \in \mathcal{M}_{k-1}^{(r)} \otimes_{A} D_{j}$ 
and write

$$
x=\sum_{I_{1}, I_{2}, \ldots, I_{r+1}} v_{I_{1} I_{2} \ldots I_{r} I_{r+1}} e_{I_{1}} \otimes e_{I_{2}} \otimes \ldots \otimes e_{I_{r}} \otimes e_{I_{r+1}} .
$$

Here every $I_{i}$ for $1 \leq i \leq r$ is a multi-index of type $\left(j_{1}, j_{2}, \ldots, j_{k-1}\right)$, where $1 \leq j_{1}<j_{2}<\ldots<j_{k-1} \leq n$, and the corresponding $e_{I_{i}}=e_{j_{1}}^{i} \wedge e_{j_{2}}^{i} \wedge$ $\ldots \wedge e_{j_{k-1}}^{i}$. Moreover, $I_{r+1}$ is a multi-index of type $\left(h_{1}, h_{2}, \ldots, h_{j}\right)$ with $1 \leq h_{1}<h_{2}<\ldots<h_{j} \leq n$ and $e_{I_{r+1}}=e_{h_{1}} \wedge e_{h_{2}} \wedge \ldots \wedge e_{h_{j}}$. This implies that

$$
\begin{aligned}
u=\partial_{k-1}^{(r)} x=\sum_{I_{1}, I_{2}, \ldots, I_{r+1}} \sum_{p_{1}, p_{2}, \ldots, p_{r}} a_{p_{1}} a_{p_{2}} \ldots a_{p_{r}} v_{I_{1} I_{2} \ldots I_{r} I_{r+1}} \\
\left(e_{p_{1}} \wedge e_{I_{1}}\right) \otimes\left(e_{p_{2}} \wedge e_{I_{2}}\right) \otimes \ldots \otimes\left(e_{p_{r}} \wedge e_{I_{r}}\right) \otimes e_{I_{r+1}} .
\end{aligned}
$$

For $i=1,2, \ldots, r$ choose any multi-indices $J_{i}$ of type $\left(j_{1}, j_{2}, \ldots, j_{l-k}\right)$, where $1 \leq j_{1}<j_{2}<\ldots<j_{k-l} \leq n$, in such a way that $J_{i}$ is a subindex of $I_{i}$, i.e. such that every index that belongs to $J_{i}$ also belongs to $I_{i}$. Next, let $e_{J_{i}}=e_{j_{1}}^{i} \wedge e_{j_{2}}^{i} \wedge \ldots \wedge e_{j_{k-l}}^{i}$. Then $e=e_{J_{1}} \otimes e_{J_{2}} \otimes \ldots \otimes e_{J_{r}}$ defines a homomorphism $\wedge e: \mathcal{M}_{s}^{(r)} \otimes_{A} D_{j} \rightarrow \mathcal{M}_{s+k-l}^{(r)} \otimes_{A} D_{j}$ for each $s$ so that the diagram

$$
\begin{array}{ccc}
\mathcal{M}_{l-1}^{(r)} \otimes_{A} D_{j-1} & \stackrel{\partial_{j-1}^{r+1}}{\longrightarrow} \mathcal{M}_{l-1}^{(r)} \otimes_{A} D_{j} & \stackrel{\partial_{l-1}^{(r)}}{\longrightarrow} \mathcal{M}_{l}^{(r)} \otimes_{A} D_{j} \\
\wedge e \downarrow & \wedge e \downarrow & \wedge e\rfloor \\
\mathcal{M}_{k-1}^{(r)} \otimes_{A} D_{j-1} \stackrel{\partial_{j-1}^{r+1}}{\longrightarrow} \mathcal{M}_{k-1}^{(r)} \otimes_{A} D_{j} \stackrel{\partial_{k-1}^{(r)}}{\longrightarrow} \mathcal{M}_{k}^{(r)} \otimes_{A} D_{j}
\end{array}
$$

is commutative. According to the construction of $e$ there must be an element $y \in \mathcal{M}_{l-1}^{(r)} \otimes_{A} D_{j}$ such that $x=y \wedge e$. By the assumption of the Proposition that $\mathcal{R}_{l j}^{(r)}=\mathcal{H}_{l j}^{(r)}$ we have $\partial_{l-1}^{(r)} y \in \operatorname{im}\left(\partial_{l-1}^{(r)} \partial_{j-1}^{r+1}\right)$, and there exists a $z \in$ $\mathcal{M}_{l-1}^{(r)} \otimes_{A} D_{j-1}$ such that $\partial_{l-1}^{(r)} \partial_{j-1}^{r+1} z=\partial_{l-1}^{(r)} y$. By the commutativity of the diagram we see that $\partial_{k-1}^{(r)} \partial_{j-1}^{r+1}(z \wedge e)=\left(\partial_{l-1}^{(r)} \partial_{j-1}^{r+1} z\right) \wedge e=\left(\partial_{l-1}^{(r)} y\right) \wedge e=$ $\partial_{k-1}^{(r)}(y \wedge e)=\partial_{k-1}^{(r)} x$, proving the assertion.

Corollary 3.2. Let $\mathbf{a}$ be an n-tuple of endomorphisms contained in the commutative subalgebra $A$ of $\operatorname{End}_{\mathcal{A}}(\mathcal{M})$.

(a) If $\mathcal{L}_{l 0}^{(r)}=\mathcal{H}_{l 0}^{(r)}$ for some $l, 0 \leq l<n$, then $\mathcal{L}_{k 0}^{(r)}=\mathcal{H}_{k 0}^{(r)}$ for all $k$, $0 \leq k \leq l$.

(b) If $\mathcal{R}_{l n}^{(r)}=\mathcal{H}_{l n}^{(r)}$ for some $l, 0<l \leq n$, then $\mathcal{R}_{k n}^{(r)}=\mathcal{H}_{k n}^{(r)}$ for all $k$, $l \leq k \leq n$.

3.3. Example 5.5 will show that the opposite of this Corollary is not true in general. However, we will also show that in some cases, and in particular in 
the case of an $\mathcal{A}$-module $\mathcal{M}$ which is both Artinian and Noetherian, equality of the vertical and the vertical-diagonal cohomology modules at $\mathcal{M}_{0}^{(r)}$, i.e. at the top left corner of Fig. 1, implies their equality at $\mathcal{M}_{n-1}^{(r)}$. Thus, this condition imposed on the cohomology modules at some point $\mathcal{M}_{k}^{(r)}$, where $0 \leq k<n$, implies that the same condition holds for all $k$. Similar remarks apply to the case of the cohomology modules on the right-most vertical of Fig. 1.

\section{Ascent and Descent.}

In the following we shall be mainly interested in the cohomology modules at the left most and the right most verticals of the double complexes $\mathcal{M}^{(r)} \otimes_{A} D$, that is in the situation of Corollary 3.2. Throughout the modules $\mathcal{M}_{n}^{(r)}$ and $\mathcal{M}_{0}^{(r)}$ will be identified with $\mathcal{M}$ and $\mathfrak{a}$ is the ideal in $A$ generated by $\mathbf{a}$.

Proposition 4.1. If $\mathcal{L}_{n-1,0}^{(r)}=\mathcal{H}_{n-1,0}^{(r)}$ for some index $r \geq 1$, then

(a) $\operatorname{ker}\left(\partial_{0}^{r+l} \ldots \partial_{0}^{r+1} \partial_{n-1}^{(r)}\right)=\operatorname{ker}\left(\partial_{n-1}^{(r)}\right)$ for all $l \geq 1$;

(b) $\operatorname{ker} \partial_{0}^{(r)} \cap \operatorname{im} \partial_{n-1}^{(r)}=0$, i.e. $\operatorname{Hom}_{A}\left(A / \mathfrak{a}^{r}, \mathcal{M}\right) \cap \mathfrak{a}^{r} \mathcal{M}=0$;

(c) $\mathcal{L}_{n-1,0}^{(s)}=\mathcal{H}_{n-1.0}^{(s)}$ for all indices $s \geq r$.

Proof. (a) The case $l=1$ is just our assumption. Assume the claim true for a certain index $l-1$ and observe that $\operatorname{ker}\left(\partial_{0}^{r+l} \ldots \partial_{0}^{r+1} \partial_{n-1}^{(r)}\right) \supseteq \operatorname{ker} \partial_{n-1}^{(r)}$, so that we only have to show that in fact the opposite inclusion holds. To this end pick $x \in \mathcal{M}_{n-1}^{(r)}$ such that $\partial_{0}^{r+l} \ldots \partial_{0}^{r+1} \partial_{n-1}^{(r)} x=0$ and write

$$
y=\partial_{0}^{r+l-1} \ldots \partial_{0}^{r+1} x=\sum_{i_{1}, i_{2}, \ldots, i_{l-1}} x_{i_{1} i_{2} \ldots i_{l-1}} e_{i_{1}}^{r+1} \otimes e_{i_{2}}^{r+2} \otimes \ldots \otimes e_{i_{l-1}}^{r+l-1} .
$$

Clearly, $x_{i_{1} i_{2} \ldots i_{l-1}} \in \mathcal{M}_{n-1}^{(r)}$, and since $\partial_{0}^{i}$ commutes with $\partial_{0}^{j}$ for $i \neq j$ we see that $\partial_{0}^{r+l} \partial_{n-1}^{(r)} y=0$. This forces $\partial_{n-1}^{(r)} y=0$ by the assumption in the statement of our proposition. Since the maps $\partial_{0}^{i}$ commute with $\partial_{n-1}^{(r)}$, we conclude that $\partial_{0}^{r+l-1} \ldots \partial_{0}^{r+1} \partial_{n-1}^{(r)} x=0$ and the assertion follows by the induction hypothesis.

(b) The homomorphism $\partial_{n-1}^{(r)}$ is a composite of maps of the type $\partial_{n-1}^{i}$ for $1 \leq i \leq r$. After identifying $\mathcal{M}_{0}^{(r)}$ with $\mathcal{M}_{n-1}^{(r)}$ we may write $\partial_{0}^{(r)}=$ $\partial_{0}^{2 r} \partial_{0}^{2 r-1} \ldots \partial_{0}^{r+1}$. If $x \in \operatorname{ker} \partial_{0}^{(r)} \cap \operatorname{im} \partial_{n-1}^{(r)}$ then by assumption $x=\partial_{n-1}^{(r)} y$ for some $y \in \mathcal{M}_{n-1}^{(r)}$ and $\partial_{0}^{2 r} \partial_{0}^{2 r-1} \ldots \partial_{0}^{r+1} x=\partial_{0}^{(r)} x=0$. Consequently $\partial_{0}^{2 r} \partial_{0}^{2 r-1} \ldots \partial_{0}^{r+1} \partial_{n-1}^{(r)} y=0$ and it follows by (a) that $x=\partial_{n-1}^{(r)} y=0$. 
(c) Choose any $x \in \operatorname{ker}\left(\partial_{n-1}^{(r+1)} \partial_{0}^{r+2}\right)$ and observe that it suffices to show that $x \in \operatorname{ker} \partial_{n-1}^{(r+1)}$. Recall that $\partial_{n-1}^{(r+1)}=\partial_{n-1}^{r+1} \partial_{n-1}^{(r)}$ and that the two homomorphisms commute. We see that $y=\partial_{n-1}^{r+1} x \in \operatorname{ker}\left(\partial_{n-1}^{(r)} \partial_{0}^{r+2}\right)$ and hence by assumption $y \in \operatorname{ker} \partial_{n-1}^{(r)}$. This finally shows that $0=\partial_{n-1}^{(r)} \partial_{n-1}^{r+1} x=$ $\partial_{n-1}^{(r+1)} x$.

Proposition 4.2. If $\mathcal{R}_{1 n}^{(r)}=\mathcal{H}_{1 n}^{(r)}$ for some index $r \geq 1$, then

(a) $\operatorname{im}\left(\partial_{n-1}^{r+l} \ldots \partial_{n-1}^{r+1}\right) \partial_{0}^{(r)}=\operatorname{im} \partial_{0}^{(r)}$ for all $l \geq 1$;

(b) $\operatorname{ker} \partial_{0}^{(r)}+\operatorname{im} \partial_{n-1}^{(r)}=\mathcal{M}$, i.e. $\operatorname{Hom}_{A}\left(A / \mathfrak{a}^{r}, \mathcal{M}\right)+\mathfrak{a}^{r} \mathcal{M}=\mathcal{M}$;

(c) $\mathcal{R}_{1 n}^{(s)}=\mathcal{H}_{1 n}^{(s)}$ for all indices $s \geq r$.

Proof. (a) The case $l=1$ is identical with our assumption. Suppose that the claim is true for an index $l-1$ and observe that $\operatorname{im}\left(\partial_{n-1}^{r+l} \ldots \partial_{n-1}^{r+1}\right) \partial_{0}^{(r)} \subseteq$ $\operatorname{im} \partial_{0}^{(r)}$, so that it suffices to show that the opposite inclusion holds. To this end pick $x \in \mathcal{M}_{1}^{(r)} \otimes_{A} D_{n}$ such that $x \in \operatorname{im} \partial_{0}^{(r)}$ and assume by the induction hypothesis that $x=\left(\partial_{n-1}^{r+l} \ldots \partial_{n-1}^{r+2}\right) \partial_{0}^{(r)} y$ for some $y \in \mathcal{M}_{0}^{(r)} \otimes_{A}^{l-1} D_{n-1}$. Then

$$
y=\sum_{i_{2}, i_{3}, \ldots, i_{l}} y_{i_{2} i_{3} \ldots i_{l}} \hat{e}_{i_{2}}^{r+2} \otimes_{A} \hat{e}_{i_{3}}^{r+3} \otimes_{A} \cdots \otimes_{A} \hat{e}_{i_{l}}^{r+l},
$$

where the exterior products $\hat{e}_{i}^{j}=\wedge_{p \neq i} e_{p}^{j}$ form the canonical basis elements of $D_{n-1}$ and where $y_{i_{2} i_{3} \ldots i_{l}} \in \mathcal{M}_{0}^{(r)} \cong \mathcal{M}_{0}^{(r)} \otimes_{A} D_{n}$. Clearly, $\partial_{0}^{(r)} y_{i_{2} i_{3} \ldots i_{l}} \in$ $\mathcal{M}_{1}^{(r)} \otimes_{A} D_{n}$, so that by the assumption in our proposition there is an element $z_{i_{2} i_{3} \ldots i_{l}} \in \mathcal{M}_{0}^{(r)} \otimes_{A} D_{n-1}$ such that $\partial_{0}^{(r)} y_{i_{2} i_{3} \ldots i_{l}}=\partial_{n-1}^{r+1} \partial_{0}^{(r)} z_{i_{2} i_{3} \ldots i_{l}}$. Since the maps $\partial_{n-1}^{i}$ and $\partial_{n-1}^{j}$ commute for $i \neq j$, we conclude for

$$
z=\sum_{i_{2}, i_{3}, \ldots, i_{l}} z_{i_{2} i_{3} \ldots i_{l}} \hat{e}_{i_{1}}^{r+2} \otimes_{A} \hat{e}_{i_{2}}^{r+3} \otimes_{A} \ldots \otimes_{A} \hat{e}_{i_{l}}^{r+l}
$$

that $\left(\partial_{n-1}^{r+l} \ldots \partial_{n-1}^{r+1}\right) \partial_{0}^{(r)} z=x$, which proves the assertion.

(b) Choose any $x \in \mathcal{M}$. The homomorphism $\partial_{0}^{(r)}$ is a composite of maps of the type $\partial_{0}^{i}$ for $1 \leq i \leq r$. After identifying $\mathcal{M}_{0}^{(r)}$ with $\mathcal{M}_{n}^{(r)}$ we may write $\partial_{n-1}^{(r)}=\partial_{n-1}^{2 r} \partial_{n-1}^{2 r-1} \ldots \partial_{n-1}^{r+1}$. By Corollary 3.2. (b) the element $\partial_{0}^{(r)} x$ belongs to $\operatorname{im}\left(\partial_{n-1}^{(r)} \partial_{0}^{(r)}\right)$, yielding the existence of an element $y$ for which $\partial_{n-1}^{(r)} \partial_{0}^{(r)} y=\partial_{0}^{(r)} x$ and hence $\partial_{0}^{(r)}\left(x-\partial_{n-1}^{(r)} y\right)=0$.

(c) Pick any $x \in \operatorname{im} \partial_{0}^{(r+1)}$ and observe that it obviously suffices to show that $x \in \operatorname{im}\left(\partial_{0}^{(r+1)} \partial_{n-1}^{r+2}\right)$. Recall first that $\partial_{0}^{(r+1)}=\partial_{0}^{r+1} \partial_{0}^{(r)}$ and that the two maps commute. If $y$ is such that $x=\partial_{0}^{r+1} \partial_{0}^{(r)} y$ then $\partial_{0}^{r+1} y \in \operatorname{im} \partial_{0}^{(r)}=$ 
$\operatorname{im}\left(\partial_{n-1}^{r+2} \partial_{0}^{(r)}\right)$ by the induction hypothesis and by the assumption in the Proposition. Thus we conclude that $x=\partial_{0}^{(r)} \partial_{0}^{r+1} y \in \operatorname{im}\left(\partial_{n-1}^{r+2} \partial_{0}^{(r+1)}\right)$.

4.3. If there exists an index $r$ for which at the point $\mathcal{M}_{n-1}^{(r)} \otimes_{A} D_{0}$ the left vertical cohomology $\mathcal{H}_{n-1,0}^{(r)}$ is equal to the vertical-diagonal cohomology $\mathcal{L}_{n-1,0}^{(r)}$, then by Proposition 4.1 the same is true for all larger indices. The smallest index $r$ with this property will be called the ascent of the $n$-tuple a. If such an index does not exist, we shall say that the ascent of the $n$-tuple is infinite.

Similarly, if there is an index $s$ for which at the point $\mathcal{M}_{1}^{(s)} \otimes_{A} D_{n}$ the right vertical cohomology $\mathcal{H}_{1 n}^{(s)}$ is equal to the diagonal-vertical cohomology $\mathcal{R}_{1 n}^{(s)}$, then by Proposition 4.2 the same holds for all larger indices. The smallest index $s$ with this property will be called the descent of the $n$-tuple a. If such an index does not exist, we shall say that the descent of the $n$-tuple is infinite.

More generally, for any pair $(i, j)$ satisfying $0 \leq i, j \leq n$, let $d_{i j}^{0}$ be the smallest among the indices $r$ for which $\mathcal{L}_{i, j}^{(r)}=\mathcal{H}_{i, j}^{(r)}$ and $d_{i j}^{1}$ the smallest among the indices $r$ for which $\mathcal{R}_{i j}^{(r)}=\mathcal{H}_{i j}^{(r)}$. We let any of these indices be infinite whenever there is no $r$ satisfying the respective condition. According to this notation the ascent of the $n$-tuple $\mathbf{a}$ is $d_{0, n-1}^{0}$ and the descent is $d_{n 1}^{1}$.

\section{Relations between ascent, descent and Fitting's Lemma.}

In this section we will show that both ascent and descent of a commuting $n$-tuple $\mathbf{a}$ are finite if and only if a has the Fitting property and $\mathfrak{a}$ acts nilpotently on $\mathcal{K}$, and that ascent and descent are equal in this case. Let us recall from Section 4, that the notation for the ascent and the descent are $d^{0}=d_{0, n-1}^{0}$ and $d^{1}=d_{1 n}^{1}$, respectively. It is also useful to remember that $\mathcal{H}_{00}^{(r)} \cong \operatorname{Hom}_{A}\left(A / \mathfrak{a}^{r}, \mathcal{M}\right), \mathcal{H}_{n n}^{(r)} \cong \mathcal{M} / \mathfrak{a}^{r} \mathcal{M}, \mathrm{七}_{00}^{(r)}=\mathcal{H}_{00}^{(r+1)}$ and $\mathcal{R}_{n n}^{(r)}=\mathcal{H}_{n n}^{(r+1)}$.

Proposition 5.1. Let $\mathbf{a}$ be an n-tuple of endomorphisms contained in the commutative subalgebra $A$ of $\operatorname{End}_{\mathcal{A}}(\mathcal{M})$.

(a) If $\mathcal{L}_{00}^{(r)}=\mathcal{H}_{00}^{(r)}$ for some index $r \geq 1$, then $\mathcal{L}_{00}^{(s)}=\mathcal{H}_{00}^{(s)}$ for all $s \geq r$.

(b) If $\mathcal{R}_{n n}^{(r)}=\mathcal{H}_{n n}^{(r)}$ for some index $r \geq 1$, then $\mathcal{R}_{n n}^{(s)}=\mathcal{H}_{n n}^{(s)}$ for all $s \geq r$.

(c) If $\mathcal{L}_{00}^{(r)}=\mathcal{H}_{00}^{(r)}$ for a fixed index $r \geq 1$ and $\mathcal{R}_{1 n}^{(s)}=\mathcal{H}_{1 n}^{(s)}$ for some index $s \geq 1$, then this holds for some $s \leq r$.

(d) If $\mathcal{R}_{n n}^{(r)}=\mathcal{H}_{n n}^{(r)}$ for a fixed index $r \geq 1$ and $\mathcal{L}_{n-1,0}^{(s)}=\mathcal{H}_{n-1,0}^{(s)}$ for some index $s \geq 1$ then this holds for some $s \leq r$.

Proof. For (a) and (b) use similar arguments as in the proofs of Propositions 4.1(a) and 4.2(a). 
(c) Let $s$ be the smallest index for which $\mathcal{R}_{1 n}^{(s)}=\mathcal{H}_{1 n}^{(s)}$ and suppose that the assertion is false. Then, there must be an $r<s$ with $\mathcal{L}_{00}^{(r)}=\mathcal{H}_{00}^{(r)}$. By (a) we may assume with no loss of generality that $r=s-1$. We will show that in this case we must also have $\mathcal{R}_{1 n}^{(s-1)}=\mathcal{H}_{1 n}^{(s-1)}$, in contradiction to the minimality of $s$, thus proving the assertion. Notice that we always have $\operatorname{im}\left(\partial_{n-1}^{s} \partial_{0}^{(s-1)}\right) \subseteq \operatorname{im} \partial_{0}^{(s-1)}$ at the point $\mathcal{M}_{1}^{(s)} \otimes_{A} D_{n}$, so that only the opposite inclusion has to be established. For this purpose pick any $x \in \operatorname{im} \partial_{0}^{(s-1)}$. Then $x=\partial_{0}^{(s-1)} y$ for some $y \in \mathcal{M}_{0}^{(s-1)} \otimes_{A} D_{n}$ and it follows that $\partial_{0}^{s} x \in \operatorname{im} \partial_{0}^{(s)}=\operatorname{im} \partial_{0}^{(s)} \partial_{n-1}^{s+1}$ by the second assumption in (c). This yields the existence of an element $z \in \mathcal{M}_{0}^{(s)} \otimes_{A} D_{n-1}$ such that $\partial_{(0)}^{(s)} y=\partial_{0}^{s} x=$ $\partial_{0}^{(s)} \partial_{n-1}^{s+1} z$, and hence $y-\partial_{n-1}^{s+1} z \in \operatorname{ker} \partial_{0}^{(s)}$. Thus $\mathcal{H}_{00}^{(s)}=\mathcal{L}_{00}^{(s-1)}=\mathcal{H}_{00}^{(s-1)}$, where the first equality holds by definition and the second by assumption. Therefore, $x=\partial_{0}^{(s-1)} y=\partial_{0}^{(s-1)} \partial_{n-1}^{s+1} z$ which belongs to $\operatorname{im}\left(\partial_{0}^{(s-1)} \partial_{n-1}^{s+1}\right)$, establishing the inclusion $\operatorname{im} \partial_{0}^{(s-1)} \subseteq \operatorname{im}\left(\partial_{0}^{(s-1)} \partial_{n-1}^{s+1}\right)$ and hence the required contradiction.

(d) Let $s$ be the smallest index for which $\mathcal{L}_{n-1,0}^{(s)}=\mathcal{H}_{n-1,0}^{(s)}$ and suppose that the assertion is false. Then, there must be an $r<s$ such that $\mathcal{R}_{n n}^{(r)}=$ $\mathcal{H}_{n n}^{(r)}$. By (b) we may assume with no loss of generality that $r=s-1$. We shall show that in this case we must also have $\mathcal{L}_{n-1,0}^{(s-1)}=\mathcal{H}_{n-1,0}^{(s-1)}$, contradicting the minimality of $s$ and therefore proving our assertion. Recall that we always have $\operatorname{ker} \partial_{n-1}^{(s-1)} \subseteq \operatorname{ker}\left(\partial_{n-1}^{(s-1)} \partial_{0}^{s}\right)$, so that only the opposite inclusion has to be established. Pick any $x \in \operatorname{ker}\left(\partial_{n-1}^{(s-1)} \partial_{0}^{s}\right)$, then $y=\partial_{n-1}^{(s-1)} x \in$ ker $\partial_{0}^{s} \cap \operatorname{im} \partial_{n-1}^{(s-1)}$. Since $\mathcal{H}_{n n}^{(s)}=\mathcal{R}_{n n}^{(s-1)}=\mathcal{H}_{n n}^{(s-1)}$, where the first equality holds by definition and the second by assumption, it follows that $y=\partial_{n-1}^{(s)} z$ for some $z \in \mathcal{M}_{n-1}^{(s)}$ and hence $0=\partial_{0}^{s} y=\partial_{0}^{s} \partial_{n-1}^{(s)} z$. This implies, by the assumption $\mathcal{L}_{n-1,0}^{(s)}=\mathcal{H}_{n-1,0}^{(s)}$, that $y=\partial_{n-1}^{(s)} z=0$ and hence $x \in \operatorname{ker} \partial_{n-1}^{(s-1)}$, establishing the required contradiction.

Theorem 5.2. The ascent $d^{0}$ and the descent $d^{1}$ of a commuting n-tuple a are both finite if and only if $\mathcal{M}=\mathcal{K} \oplus \mathcal{I}$ as an $A$-module, where the ideal $\mathfrak{a}$ generated by the $n$-tuple a acts nilpotently on $\mathcal{K}$,

$$
\operatorname{Hom}_{A}(A / \mathfrak{a}, \mathcal{I})=0 \quad \text { and } \quad \mathfrak{a} \mathcal{I}=\mathcal{I} \text {. }
$$

If these equivalent conditions are satisfied, then

(a) $d^{0}=r=d^{1}$.

(b) $d_{j-1,0}^{0}=r=d_{j n}^{1}$ for $1 \leq r \leq n$.

(c) $\mathcal{K}=\operatorname{ker} \partial_{0}^{(r)} \cong \operatorname{Hom}_{A}\left(A / \mathfrak{a}^{r}, \mathcal{M}\right)$ and $\mathcal{I}=\operatorname{im} \partial_{n-1}^{(r)}=\mathfrak{a}^{r} \mathcal{M}$. 
(d) The restrictions of the $a_{i}$ to ker $\partial_{0}^{(r)}$ are nilpotent. Moreover,

$$
\operatorname{Hom}_{A}\left(A / \mathfrak{a}^{(k)}, \mathcal{I}\right)=0 \quad \text { and } \quad \mathfrak{a}^{(k)} \mathcal{I}=\mathcal{I}
$$

for every $k \geq 1$, where $\mathfrak{a}^{(k)}$ is the ideal in A generated by the $n$-tuple $\mathbf{a}^{(k)}=\left(a_{1}^{k}, a_{2}^{k}, \ldots, a_{n}^{k}\right)$.

(e) $r$ is an invariant of the ideal $\mathfrak{a}$, i.e. independent of the particular finite generating set $\mathbf{a}$.

Proof. Recall that the ascent is $d^{0}=d_{n-1,0}^{0}$ and the descent $d^{1}=d_{1 n}^{1}$. Suppose that they are both finite. By Propositions 4.1(c) and 5.1(c) we have that $d_{n-1,0}^{0} \geq d_{n-2,0}^{0} \geq \ldots \geq d_{0,0}^{0} \geq d_{1 n}^{1}$, proving in particular that the ascent is no smaller than the descent. On the other hand, Propositions $4.2(\mathrm{c})$ and $5.1(\mathrm{~d})$ yield $d_{1 n}^{1} \geq d_{2 n}^{1} \geq \ldots \geq d_{n n}^{1} \geq d_{n-1,0}^{0}$ and, in particular, that the descent is no smaller than the ascent. Thus all the indices and, in particular the ascent and the descent, are equal as required in (b) and in (a). Assertion (c) is an easy consequence of Propositions 4.1(b) and 4.2(b). That the decomposition is invariant under the actions of $A$ and of $\mathcal{A}$ is due to the fact that the differentials are compatible with both actions. Now, (d) follows directly from (c).

Conversely, suppose that $\mathcal{M}=\mathcal{K} \oplus \mathcal{I}$, where the restriction of the $a_{i}$ to $\mathcal{K}$ are nilpotent and the restrictions $a_{i}^{\prime}$ of the $a_{i}$ to $\mathcal{I}$ satisfy

$$
\cap_{i=1}^{n} \operatorname{ker} a_{i}^{\prime}=0 \text { and } \sum_{i=1}^{n} \operatorname{im} a_{i}^{\prime}=\mathcal{I} \text {. }
$$

Then for each $r \geq 1$ there is obviously an isomorphism of complexes

$$
\mathcal{M}^{(r)} \cong \mathcal{K}^{(r)} \oplus \mathcal{I}^{(r)},
$$

and so it suffices to prove the assertion separately for $\mathcal{K}$ and for $\mathcal{I}$.

For $\mathcal{N}$ equal to $\mathcal{K}$ or $\mathcal{I}$, as the case may be, consider first the diagram

$$
\begin{aligned}
& \mathcal{N}_{n-1}^{(r)} \\
& \partial_{n-1}^{(r)} \downarrow \\
& \mathcal{N}_{n}^{(r)} \stackrel{\partial_{n}^{r+1}}{\longrightarrow} \mathcal{N}_{n}^{(r)} \otimes_{A} D_{1} .
\end{aligned}
$$

Let $E_{i}=e_{1} \wedge \ldots \wedge \hat{e}_{i} \wedge \ldots e_{n}$. Then for $\mathcal{N}=\mathcal{K}$, we see that

$$
\begin{aligned}
& \partial_{n-1}^{(r)}\left(\sum_{i_{1}, i_{2}, \ldots, i_{r}} x_{i_{1} i_{2} \ldots i_{r}} E_{i_{1}} \otimes E_{i_{2}} \otimes \ldots \otimes E_{i_{r}}\right) \\
& =\sum_{i_{1}, i_{2}, \ldots, i_{r}} a_{i_{1}} a_{i_{2}} \ldots a_{i_{r}} x_{i_{1} i_{2} \ldots i_{r}}=0
\end{aligned}
$$

if $r$ is large enough, since each coefficient $x_{i_{1} i_{2} \ldots i_{r}}$ is in $\mathcal{K}$. It suffices to choose $r \geq \sum_{i} r_{i}$, where $a_{i}^{r_{i}} \mathcal{K}=0$. Thus, there is an index $r_{0}$, such that $\partial_{n-1}^{(r)}=0$ 
for all $r \geq r_{0}$, and $(\mathbf{a}, \mathcal{K})$ has finite ascent. On the other hand, when $\mathcal{N}=\mathcal{I}$ then

$$
\partial_{n}^{r+1}(y)=\sum_{j} a_{j}^{\prime} y \otimes e_{j}=0
$$

if and only if $y=0$, since $y \in \mathcal{I}$ and $\cap_{j} \operatorname{ker} a_{j}^{\prime}=0$. Hence, $\partial_{n}^{r+1}$ is injective for every $r$ and $\left(\mathbf{a}^{\prime}, \mathcal{I}\right)$ has finite ascent. Now consider the diagram

$$
\begin{aligned}
\mathcal{N}_{0}^{(r)} \otimes_{A} D_{n-1} \stackrel{\partial_{n-1}^{r+1}}{\longrightarrow} & \mathcal{N}^{(r)} \otimes_{A} D_{n} \\
& \downarrow \partial_{0}^{(r)} \\
& \mathcal{N}_{1}^{(r)} \otimes_{A} D_{n} .
\end{aligned}
$$

Then, setting $\mathcal{N}=\mathcal{K}$, we see that

$$
\partial_{0}^{(r)}(x)=\sum_{j_{i}, j_{2}, \ldots, j_{n}} a_{j_{1}} a_{j_{2}} \ldots a_{j_{r}} x e_{j_{1}} \otimes e_{j_{2}} \otimes \ldots \otimes e_{j_{r}}=0
$$

if $r$ is large enough, since $x \in \mathcal{K}$. Again, it suffices to choose $r \geq \sum_{i} r_{i}$, where $a_{i}^{r_{i}} \mathcal{K}=0$. Thus, there is an index $s_{0}$ such that $\partial_{0}^{(r)}=0$ for all $r \geq s_{0}$, so that $(\mathbf{a}, \mathcal{K})$ has finite descent. If $\mathcal{N}=\mathcal{I}$ then

$$
\partial_{n-1}^{r+1}\left(\sum_{i} x_{i} e_{1} \wedge \ldots \wedge \hat{e}_{i} \wedge \ldots \wedge e_{n}\right)=\sum_{i}(-1)^{i-1} a_{i}^{\prime} x_{i} .
$$

Thus, $\partial_{n-1}^{r+1}$ is surjective for every $r$, since $\mathcal{I}=\sum_{i} \operatorname{im} a_{i}^{\prime}$, so that $\left(\mathbf{a}^{\prime}, \mathcal{I}\right)$ has finite descent. The ascent and the descent of $\mathbf{a}$ are therefore both finite, hence equal. Moreover, it follows directly from our conditions, which are independent of the particular finite generating set of the ideal $\mathfrak{a}$, that this number $r$ is the least positive integer for which one and hence all the conditions $\mathfrak{a}^{r} \mathcal{K}=0, \mathcal{I}=\mathfrak{a}^{r} \mathcal{M}$ and $\mathcal{K}=\operatorname{Hom}_{A}\left(A / \mathfrak{a}^{r}, \mathcal{M}\right)$ are satisfied, so that (e) holds.

5.3. The conditions $\operatorname{Hom}_{A}(A / \mathfrak{a}, \mathcal{I})=0$ and $\mathcal{I}=\mathfrak{a} \mathcal{I}$ say that the $n$-tuple a acts 'jointly bijectively' on $\mathcal{I}$. Observe that a slight modification of the proof of Theorem 5.2 shows that ascent and descent are either both finite or both infinite whenever a has the Fitting property, i.e. whenever $\mathcal{M}=\mathcal{K} \oplus \mathcal{I}$ and $\mathcal{I}=\mathfrak{a} \mathcal{I}$, where $\mathcal{K}=\cup_{r} \operatorname{Hom}_{a}\left(A / \mathfrak{a}^{r}, \mathcal{M}\right)$ and $\mathcal{I}=\cap_{r} \mathfrak{a}^{r} \mathcal{M}$. As a more general version of assertion (e) we shall see in Section 8 that, even in the absence of the Fitting property, both ascent and descent are invariants of the ideal $\mathfrak{a}$.

We say that the commuting $n$-tuple satisfies condition (F) if the $\mathcal{A}$-module $\mathcal{M}$ decomposes into a direct sum of $\mathcal{A}$-submodules

$$
\mathcal{M}=\mathcal{K} \oplus \mathcal{I}_{1} \oplus \mathcal{I}_{2} \oplus \ldots \oplus \mathcal{I}_{p}
$$


where $\mathcal{I}_{i}$ for $0 \leq i \leq p$ are invariant under $A$, where the restrictions of the $a_{j}$ to $\mathcal{K}$ are nilpotent, while for $i=1,2, \ldots, p$ at least one of the restrictions to $\mathcal{I}_{i}$ is invertible. Observe that if $\mathcal{M}$ is both Artinian and Noetherian as an $\mathcal{A}$-module, then every commuting $n$-tuple of endomorphisms satisfies this condition. Indeed, in this case the ascent and the descent of the endomorphism $a_{1}$ must be finite by Fitting's Lemma $[\mathbf{P}, \mathbf{B}]$. By Theorem 5.2, applied to the 1-tuple $a_{1}$ and the fixed commutative algebra $A$, the $\mathcal{A}$-module $\mathcal{M}$ decomposes into $\mathcal{M}=\mathcal{K}_{1} \oplus \mathcal{I}_{1}$, where $\mathcal{K}_{1}=\operatorname{ker} a_{1}^{r}$ and $\mathcal{I}_{1}=\operatorname{im} a_{1}^{r}$ for some sufficiently large exponent $r$. By commutativity, $\mathcal{K}_{1}$ and $\mathcal{I}_{1}$ are $A$-invariant. If the restrictions of all the $a_{i}$ to $\mathcal{K}_{1}$ are nilpotent, then we are done. If not, pick one whose restriction to $\mathcal{K}_{1}$ is not nilpotent, say $a_{2}$, and decompose $\mathcal{K}_{1}$ into a direct sum of $\mathcal{A}$-submodules $\mathcal{K}_{2} \oplus \mathcal{I}_{2}$ as above. Obviously, the inductive procedure must terminate after finitely many steps and we get the desired decomposition. These considerations actually show slightly more. In order that condition $(\mathrm{F})$ is satisfied it suffices that each member of the $n$-tuple a has finite ascent and finite descent.

Corollary 5.4. If each member of the n-tuple a has finite ascent and finite descent, in particular, when the $\mathcal{A}$-module $\mathcal{M}$ is both Artinian and Noetherian, then both ascent and descent of the $n$-tuple are finite and the conclusions of Theorem 5.2 are valid.

Proof. The "Fitting" decomposition of $\mathcal{M}$, given in condition (F) and guaranteed by our assumptions, decomposes $\mathcal{M}_{n-1}^{(r)}=\mathcal{M} \otimes_{A} D_{n-1}^{(r)}$ into

$$
\mathcal{M}_{n-1}^{(r)}=\left(\mathcal{K} \otimes_{A} D_{n-1}^{(r)}\right) \oplus\left(\mathcal{I}_{1} \otimes_{A} D_{n-1}^{(r)}\right) \oplus \cdots \oplus\left(\mathcal{I}_{p} \otimes_{A} D_{n-1}^{(r)}\right) .
$$

An element $x \in \operatorname{ker}\left(\partial_{0}^{r+1} \partial_{n-1}^{(r)}\right)$ decomposes accordingly into $x=u_{0}+u_{1}+$ $\cdots+u_{p}$. Since the differentials $\partial_{n-1}^{(r)}$ and $\partial_{0}^{r+1}$ preserve the decomposition it follows that

$$
0=\partial_{0}^{r+1} \partial_{n-1}^{(r)} x=\partial_{0}^{r+1} \partial_{n-1}^{(r)} u_{0}+\partial_{0}^{r+1} \partial_{n-1}^{(r)} u_{1}+\cdots+\partial_{0}^{r+1} \partial_{n-1}^{(r)} u_{0} .
$$

For $0 \leq i \leq p$ the term $\partial_{n-1}^{(r)} u_{i}$ must therefore be in the kernel of each $a_{j}$ restricted to $\mathcal{K}$ and $\mathcal{I}_{i}$, respectively. This forces $\partial_{n-1}^{(r)} u_{i}$ to be zero for $1 \leq i \leq p$, since then at least one of these restrictions is invertible. Hence $\partial_{n-1}^{(r)} x=\partial_{n-1}^{(r)} u_{0}$, and by the nilpotency of the restriction of each $a_{j}$ to $\mathcal{K}$ we see that $\partial_{n-1}^{(r)} x=\partial_{n-1}^{(r)} u_{0}=0$ for $r$ sufficiently large. Similarly, if $y=$ $\partial_{0}^{(r)} x \in \operatorname{im} \partial_{0}^{(r)}$, decompose $x$ according to (F) into $x=u_{0}+u_{1}+\cdots+u_{p}$. For sufficiently large $r$ we may assume $u_{0}=0$ with no loss of generality. But then $x \in \operatorname{im} \partial_{n-1}^{r+1}$, since the restriction $\partial_{n-1}: \oplus_{i=1}^{p} \mathcal{I}_{i} \otimes_{A} D_{n-1} \rightarrow \oplus_{i=1}^{p} \mathcal{I}_{i} \otimes_{A} D_{n}$ is surjective.

Examples 5.5. a) The converse of Corollary 5.4 is false. Here is an example. Let $V=\mathcal{F}(N, F)$ be the vector space of all sequences in the field $F$. Consider 
the pair of commuting linear operators $\mathbf{a}=(a, b)$ on $V$ defined by

$$
(a f)(n)=\left\{\begin{array}{ll}
0, & \text { for } n=1 \\
f(n), & \text { for } n>1
\end{array} \text { and }(b f)(n)=\left\{\begin{array}{ll}
0, & \text { for } n=1 \\
f(n+1), & \text { for } n>1
\end{array} .\right.\right.
$$

Since $a$ is idempotent, $\operatorname{ker} a^{s}=\operatorname{ker} a=\{f \mid f(n)=0$ for $n>1\}$ and $\operatorname{im} a^{s}=$ $\operatorname{im} a=\{f \mid f(1)=0\}$, we see that the ascent and the descent of $a$ are both equal to 1 . On the other hand, $\operatorname{ker} b^{r}=\{f \mid f(n)=0$ for $n \geq r+2\}$ and $\operatorname{im} b^{r}=\operatorname{im} b=\{f \mid f(1)=0\}$, so that $b$ has infinite ascent but descent 1 .

b) There is a pair of commuting endomorphisms on an infinite dimensional vector space $\mathcal{M}$ for which the ascent $d^{0}=d_{n-1,0}^{0}$ is infinite and the index $d_{00}^{0}$ is finite.

Construction. Let $F$ be an arbitrary field and let $\mathcal{M}$ be the vector space of pairs $(\alpha, \mathbf{p})$, where $\alpha \in F$ and $\mathbf{p}=\left(p_{i}(x)\right)$ is a sequence of polynomials with coefficients in $F$. If we define $a: \mathcal{M} \rightarrow \mathcal{M}$ by

$$
a\left(\alpha, p_{1}(x), p_{2}(x), \ldots\right)=\left(0, x p_{1}(x), x p_{2}(x), \ldots\right)
$$

and $b: \mathcal{M} \rightarrow \mathcal{M}$ by

$$
b\left(\alpha, p_{1}(x), p_{2}(x), \ldots\right)=\left(p_{1}(0), p_{2}(x), p_{3}(x), \ldots\right)
$$

then it is easy to verify that $a$ and $b$ are commuting endomorphisms of $\mathcal{M}$. Moreover, we see that $a^{n}\left(\alpha, p_{1}(x), p_{2}(x), \ldots\right)=\left(0, x^{n} p_{1}(x), x^{n} p_{2}(x), \ldots\right)$ and hence $\operatorname{ker} a^{n}=\{(\alpha, 0)\}$ for all $n \geq 1$. Since ker $a \subset \operatorname{ker} b$, we must necessarily have $\cap_{i+j=n} \operatorname{ker}\left(a^{i} b^{j}\right)=\operatorname{ker} a$ for all $n \geq 1$, showing that $d_{00}^{0}=1$. In order to see that the ascent of this pair is infinite, choose for $r \geq 1$ an element $u=(\alpha, \mathbf{p}) \in \mathcal{M}$ such that $p_{r}(0) \neq 0$ and $p_{k}(x)=0$ for $k>r$. It then follows that $0 \neq b^{r} u=\left(p_{r}(0), 0,0, \ldots\right) \in \operatorname{ker} a$. Let $\hat{u}=u \hat{e}_{1}^{1} \otimes \hat{e}_{1}^{2} \otimes \ldots \otimes \hat{e}_{1}^{r} \in$ $\mathcal{M}_{n-1}^{(r)}$. Then $b^{r} u=\partial_{n-1}^{(r)} \tilde{u}$ is a non-zero vector from ker $\partial_{0}^{r+1}=\operatorname{ker} a$. Hence, $\operatorname{ker} \partial_{n-1}^{(r)} \neq \operatorname{ker}\left(\partial_{0}^{r+1} \partial_{n-1}^{(r)}\right)$ for every $r \geq 1$.

\section{Some remarks and comparisons.}

6.1. If $\mathfrak{a}$ is the ideal generated by $\mathbf{a}=\left(a_{1}, a_{2}, \ldots, a_{n}\right)$ and $\mathfrak{a}^{(s)}$ the ideal generated by $\mathbf{a}^{s}=\left(a_{1}^{s}, a_{2}^{s}, \ldots, a_{n}^{s}\right)$ in $A$ then clearly $\mathfrak{a}^{(n s)} \subseteq \mathfrak{a}^{n s} \subseteq \mathfrak{a}^{(s)} \subseteq \mathfrak{a}^{s}$, $\varliminf_{j} \operatorname{Hom}_{A}\left(A / \mathfrak{a}^{j}, \mathcal{M}\right)=\varliminf_{j} \operatorname{Hom}_{A}\left(A / \mathfrak{a}^{(j)}, \mathcal{M}\right)$ and $\cap_{j} \mathfrak{a}^{j} \mathcal{M}=\cap_{j} \mathfrak{a}^{(j)} \mathcal{M}$. The local cohomology with coefficients in the $A$-module $\mathcal{M}$ of the ideal $\mathfrak{a}$ is defined by

$$
H_{\mathfrak{a}}^{*}(\mathcal{M})=\varliminf_{\lim _{s}} \operatorname{Ext}_{A}^{*}\left(A / \mathfrak{a}^{s}, \mathcal{M}\right) .
$$

An equivalent description in terms of Koszul complexes can be given as follows. For each $s \geq 1$ the maps

$$
\chi: K^{*}\left(M, \mathbf{a}^{s}\right) \rightarrow K^{*}\left(M, \mathbf{a}^{s+1}\right) \quad \text { and } \quad \eta: K^{*}\left(M, \mathbf{a}^{s+1}\right) \rightarrow K^{*}\left(M, \mathbf{a}^{s}\right),
$$


defined by $\chi\left(m e_{i_{1}} \wedge e_{i_{2}} \wedge \ldots \wedge e_{i_{l}}\right)=a_{i_{1}} a_{i_{2}} \ldots a_{i_{l}} m e_{i_{1}} \wedge e_{i_{2}} \wedge \ldots \wedge e_{i_{l}}$ and by $\eta\left(m e_{i_{1}} \wedge e_{i_{2}} \wedge \ldots \wedge e_{i_{l}}\right)=a_{i_{l+1}} a_{i_{l+2}} \ldots a_{i_{n}} m e_{i_{1}} \wedge e_{i_{2}} \wedge \ldots \wedge e_{i_{l}}$, respectively, are cochain maps. It is known [BH, Theorem 3.5.6] that

$$
H_{\mathfrak{a}}^{*}(\mathcal{M})=H^{*}\left(\lim _{s} K^{*}\left(\mathcal{M}, \mathbf{a}^{s}\right)\right)=\lim _{s} H^{*}\left(K^{*}\left(\mathcal{M}, \mathbf{a}^{s}\right)\right)
$$

and in particular,

$$
H_{\mathfrak{a}}^{0}(\mathcal{M})=\underline{\lim }_{j} \operatorname{Hom}_{A}\left(A / \mathfrak{a}^{j}, \mathcal{M}\right)=\underline{\lim }_{j} \operatorname{Hom}_{A}\left(A / \mathfrak{a}^{(j)}, \mathcal{M}\right),
$$

where the direct limit is taken along the maps $\chi$.

We may also take the inverse limit along the maps $\eta$ and get a commutative diagram with exact rows

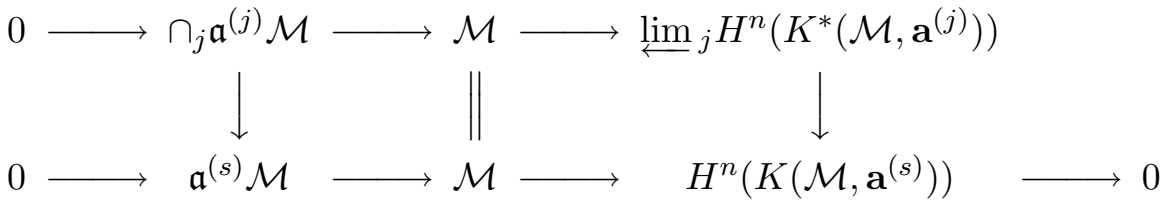

for each $s \geq 1$.

6.2. With the notation already used earlier we also have

$$
\begin{aligned}
\mathcal{K} & =\varliminf_{\lim _{j}} \operatorname{Hom}_{A}\left(A / \mathfrak{a}^{j}, \mathcal{M}\right)=\underline{\lim }_{j} \operatorname{Hom}_{A}\left(A / \mathfrak{a}^{(j)}, \mathcal{M}\right), \\
\mathcal{I} & =\cap_{j} \mathfrak{a}^{j} \mathcal{M}=\cap_{j} \mathfrak{a}^{(j)} \mathcal{M}
\end{aligned}
$$

and we get the obvious commutative diagram with exact rows

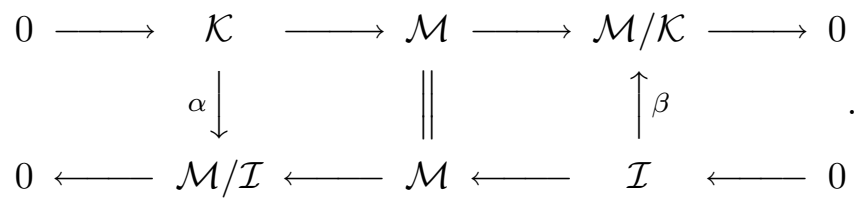

It is easy to see that:

(1) $\mathcal{K} \cap \mathcal{I}=0$ iff $\alpha$ is injective iff $\beta$ is injective;

(2) $\mathcal{K}+\mathcal{I}=\mathcal{M}$ iff $\alpha$ is surjective iff $\beta$ is surjective;

(3) $\mathcal{K} \oplus \mathcal{I}=\mathcal{M}$ iff $\alpha$ is bijective iff $\beta$ is bijective.

The ascending chain of submodules

$\operatorname{Hom}_{A}(A / \mathfrak{a}, \mathcal{M}) \subseteq \ldots \subseteq \operatorname{Hom}_{A}\left(A / \mathfrak{a}^{j}, \mathcal{M}\right) \subseteq \operatorname{Hom}_{A}\left(A / \mathfrak{a}^{j+1}, \mathcal{M}\right) \subseteq \ldots$

of $\mathcal{M}$ becomes stationary if and only if the ascending chain

$\operatorname{Hom}_{A}(A / \mathfrak{a}, \mathcal{M}) \subseteq \ldots \subseteq \operatorname{Hom}_{A}\left(A / \mathfrak{a}^{(j)}, \mathcal{M}\right) \subseteq \operatorname{Hom}_{A}\left(A / \mathfrak{a}^{(j+1)}, \mathcal{M}\right) \subseteq \ldots$

becomes stationary. It follows from Proposition 4.1 that this happens at stage $s$ when the ascent of $\mathbf{a}$ is finite and equal to $s$; in that case

$$
\operatorname{Hom}_{A}\left(A / \mathfrak{a}^{j}, \mathcal{M}\right)=\operatorname{Hom}_{A}\left(A / \mathfrak{a}^{(j)}, \mathcal{M}\right)
$$


and $\operatorname{Hom}_{A}\left(A / \mathfrak{a}^{j}, \mathcal{M}\right) \cap \mathfrak{a}^{j} \mathcal{M}=0$ for all $j \geq s$. The descending chain of submodules

$$
\mathcal{M} \supseteq \mathfrak{a} \mathcal{M} \ldots \supseteq \mathfrak{a}^{j} \mathcal{M} \supseteq \mathfrak{a}^{j+1} \mathcal{M} \supseteq \ldots
$$

becomes stationary if and only if the descending chain

$$
\mathcal{M} \supseteq \mathfrak{a} \mathcal{M} \ldots \supseteq \mathfrak{a}^{(j)} \mathcal{M} \supseteq \mathfrak{a}^{(j+1)} \mathcal{M} \supseteq \ldots
$$

becomes stationary. By Proposition 4.2 this happens at stage $r$ when the descent of $\mathbf{a}$ is finite and equal to $r$; in that case

$$
\mathfrak{a}^{j} \mathcal{M}=\mathfrak{a}^{(j)} \mathcal{M}
$$

and $\operatorname{Hom}_{A}\left(A / \mathfrak{a}^{j}, \mathcal{M}\right)+\mathfrak{a}^{j} \mathcal{M}=\mathcal{M}$ for all $j \geq r$. In each case the two chains may not terminate at the same stage as the following example shows. If $a_{1}$ and $a_{2}$ are the linear operators defined by

$$
a_{1}\left(x_{1}, x_{2}, x_{3}, x_{4}\right)=\left(x_{3}, x_{4}, 0,0\right) \quad \text { and } \quad a_{2}\left(x_{1}, x_{2}, x_{3}, x_{4}\right)=\left(x_{2}, 0, x_{4}, 0\right)
$$

on the vector space $\mathcal{M}=R^{4}$, then $a_{1}^{2}=0=a_{2}^{2}$ but $a_{1} a_{2}\left(x_{1}, x_{2}, x_{3}, x_{4}\right)=$ $\left(x_{4}, 0,0,0\right)$, so that in each case the second chain terminates at stage two and the first at stage three.

If both the ascent and the descent of $\mathbf{a}$ are finite then by Theorem 5.2 they are equal, $\mathcal{K}=\operatorname{Hom}_{A}\left(A / \mathfrak{a}^{s}, \mathcal{M}\right)=\operatorname{Hom}_{A}\left(A / \mathfrak{a}^{(s)}, \mathcal{M}\right), \mathcal{I}=\mathfrak{a}^{s} \mathcal{M}=\mathfrak{a}^{(s)} \mathcal{M}$ and we have the Fitting decomposition $\operatorname{Hom}_{A}\left(A / \mathfrak{a}^{s}, \mathcal{M}\right) \oplus \mathfrak{a}^{s} \mathcal{M}=\mathcal{M}$.

However, Example 5.5. b) shows that the stationarity of the above chains of submodules of $\mathcal{M}$ alone will not guarantee a Fitting type decomposition of $\mathcal{M}$. Therefore stationarity of these chains alone will not suffice to get a useful definition for finite ascent and finite descent.

\section{Comparison maps.}

7.1. Let $\mathbf{a}=\left(a_{1}, a_{2}, \ldots, a_{n}\right)$ be an $n$-tuple of commuting endomorphisms of the $\mathcal{A}$-module $\mathcal{M}$ and let $\mathbf{b}=\left(b_{1}, b_{2}, \ldots, b_{m}\right)$ be an $m$-tuple of commuting endomorphisms of the $\mathcal{A}$-module $\mathcal{N}$. A map of differential graded modules

$$
u: K\left(\mathcal{M}, \partial_{\mathbf{a}}\right) \rightarrow K\left(\mathcal{N}, \partial_{\mathbf{b}}\right)
$$

must satisfy the equation $\partial_{\mathbf{b}} u=u \partial_{\mathbf{a}}$, i.e. the diagram

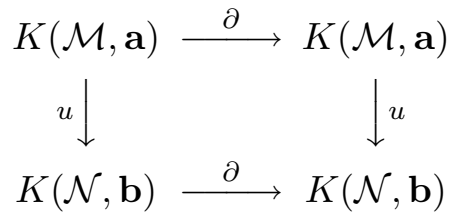

commutes. In particular, if $u_{0}: \mathcal{M} \rightarrow \mathcal{N}$ is an $\mathcal{A}$-module map and $U=\left[u_{i j}\right]$ is an $m \times n$-matrix of $\mathcal{A}$-endomorphisms of $\mathcal{N}$ such that entries from different 
rows commute, then a map of graded modules $u=\left(u_{0}, U\right): K(\mathcal{M}, \mathbf{a}) \rightarrow$ $K(\mathcal{N}, \mathbf{b})$ can be defined by

$$
\begin{aligned}
& u\left(v e_{j_{1}} \wedge e_{j_{2}} \wedge \ldots \wedge e_{j_{p}}\right) \\
& =\sum_{i_{1}, i_{2}, \ldots, i_{p}} u_{i_{1} j_{1}} u_{i_{2} j_{2}} \ldots u_{i_{p} j_{p}} u_{0}(v) f_{i_{1}} \wedge f_{i_{2}} \wedge \ldots \wedge f_{i_{p}} \\
& =\sum_{I \in J(p)} \operatorname{det}\left(\left[u_{i_{k} j_{l}}\right]_{k, l=1}^{p}\right) u_{0}(v) f_{i_{1}} \wedge f_{i_{2}} \wedge \ldots \wedge f_{i_{p}},
\end{aligned}
$$

where $J(p)=\left\{I=\left(i_{1}, i_{2}, \ldots, i_{p}\right) \mid 1 \leq i_{1}<i_{2}<\ldots<i_{p} \leq n\right\}$. Using multi-indices this can be written as

$$
u\left(v e_{J}\right)=\sum_{I \in J(p)} u_{I J} u_{0}(v) f_{I},
$$

where $u_{I J}=\operatorname{det}\left(\left[u_{i_{k} j_{l}}\right]_{k, l=1}^{p}\right)$. The following result is essentially [R, Lemma 2.1], where the condition that $u_{i j}$ and $b_{k}$ commute for $i \neq k$ should be added.

Lemma 7.2. If the diagram

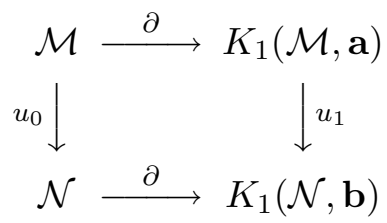

commutes, and if $u_{i j}$ commutes with $u_{k l}$ and with $b_{k}$ for $i \neq k$, then

$$
u=\left(u_{0}, U\right): K\left(\mathcal{M}, \partial_{\mathbf{a}}\right) \rightarrow K\left(\mathcal{N}, \partial_{\mathbf{b}}\right)
$$

is a cochain map. If, in addition, $u_{0}$ is an isomorphism and the matrix $\left[u_{i j}\right]$ is invertible then $u$ is an isomorphism of complexes.

Proof. The commutativity of the diagram says that

$$
\sum_{i} b_{i} u_{0}(v) f_{i}=\partial u_{0}(v)=u_{1} \partial(v)=\sum_{i, j} u_{i j} u_{0}\left(a_{j} v\right) f_{i}
$$

for every $v \in \mathcal{M}$ and hence $b_{i} u_{0}=\sum_{i} u_{i j} u_{0} a_{j}$ for $1 \leq i \leq m$. But then

$$
\begin{aligned}
& u \partial\left(v e_{j_{1}} \wedge \ldots \wedge e_{j_{p}}\right) \\
& =\sum_{j_{0}, i_{0}, I} u_{i_{0} j_{0}} u_{i_{1}, j_{1}} \ldots u_{i_{p}, j_{p}} u_{0}\left(a_{j_{0}} v\right) f_{i_{0}} \wedge f_{i_{1}} \wedge \ldots \wedge f_{i_{p}} \\
& =\sum_{i_{0}, I} b_{i_{0}} u_{i_{1}, j_{1}} \ldots u_{i_{p}, j_{p}} u_{0}(v) f_{i_{0}} \wedge f_{i_{1}} \wedge \ldots \wedge f_{i_{p}, j_{p}}=\partial u\left(v e_{J}\right),
\end{aligned}
$$


if $u_{i j}$ and $b_{k}$ commute for $i \neq k$, so that the diagram

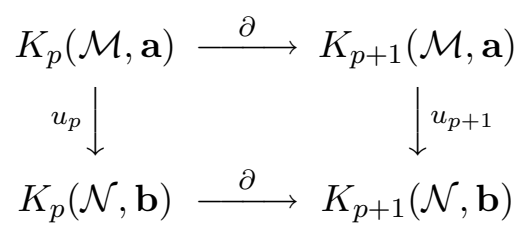

commutes for every $p \geq 0$. In terms of multi-indices this reads

$$
\begin{aligned}
u \partial\left(v e_{J}\right) & =u\left(\sum_{j} a_{j} v e_{j} \wedge e_{J}\right)=\sum_{j, i, I} u_{i j} u_{I J} u_{0}\left(a_{j} v\right) f_{i} \wedge f_{I} \\
& =\sum_{I, j, i} u_{I J} u_{i j} u_{0}\left(a_{j} v\right) f_{i} \wedge f_{I} \\
& =\sum_{i, I} b_{i} u_{I J} u_{0}(v) f_{i} \wedge f_{I}=\partial u\left(v e_{J}\right) .
\end{aligned}
$$

If, in addition, $u_{0}$ and $\left[u_{i j}\right]$ are invertible then a direct calculation shows that $\left(u_{0}^{-1},\left[u_{0}^{-1} u_{i j} u_{0}\right]^{-1}\right)=u^{-1}: K(\mathcal{N}, \mathbf{b}) \rightarrow K(\mathcal{M}, \mathbf{a})$ is the inverse of $u$.

It is the following special case of the above situation that is of particular importance for us.

Proposition 7.3. Let $A$ and $B$ be commutative $R$-subalgebras of $\operatorname{End}_{\mathcal{A}}(\mathcal{M})$ containing the $n$-tuples $\mathbf{a}$ and $\mathbf{b}$, respectively, such that $\mathbf{b} u_{0}=U u_{0} \mathbf{a}$ for some $n \times n$ matrix $U=\left[u_{i j}\right]$ of $\mathcal{A}$-endomorphisms and some $\mathcal{A}$-endomorphism $u_{0}$ of $\mathcal{M}$. If $u_{i j}$ commutes with $u_{k l}$ and with $b_{k}$ for $k \neq i$, then $U$ induces a cochain map

$$
u=\left(u_{0}, U\right): K\left(\mathcal{M}, \partial_{\mathbf{a}}\right) \rightarrow K\left(\mathcal{M}, \partial_{\mathbf{b}}\right) .
$$

Moreover, if $U$ is invertible and $u_{0}$ is an automorphism then $u$ is an isomorphism.

7.4. As the first of the following example shows, the conditions of Proposition 7.3 are not sufficient to guarantee that $\mathbf{a}$ and $\mathbf{b}$ have the same ascent or the same descent. In case $\mathcal{M}=R^{2}$ consider the matrices

$$
a=\left(\begin{array}{ll}
0 & 1 \\
0 & 0
\end{array}\right), \quad b=\left(\begin{array}{ll}
0 & 1 \\
0 & 1
\end{array}\right), \quad c=\left(\begin{array}{ll}
-1 & 1 \\
-1 & 1
\end{array}\right), \quad u=\left(\begin{array}{ll}
1 & 0 \\
1 & 1
\end{array}\right)
$$

in $\mathrm{M}_{2}(R)$. Then $b=u a, a^{2}=0, b^{2}=b$ and $c=u a u^{-1}$. Thus, with $\left(u_{0}, U\right)=(i d, u)$ the conditions of Proposition 7.3 are met, but the ascent and the descent of $a$ are both equal to 2, while those of $b$ are both equal to 1 . On the other hand, the conditions are met again if we replace $b$ by $c=u a u^{-1}$ and take $\left(u_{0}, U\right)=(u, i d)$. As expected, $a$ and $c$ have of course the same ascent and the same descent.

This, and considerations of how $\left(u_{0}, U\right)$ could induce a map of complexes from $\mathcal{M}^{(r)}(\mathbf{a})$ to $\mathcal{M}^{(r)}(\mathbf{b})$ for $r \geq 2$, suggest that what is needed to guarantee 
equal ascent and equal descent, aside from $u_{0}$ being invertible, is that the entries of $U$ commute pairwise and that they commute with the entries of b. It is obvious that ascent and descent do not depend on the particular subalgebra $A$ of $\operatorname{End}_{\mathcal{A}}(\mathcal{M})$ containing a. If $u_{0} \in \operatorname{Aut}_{\mathcal{A}}(\mathcal{M})$ then $\mathbf{b} u_{0}=$ $U u_{0} \mathbf{a}$ is equivalent to $\mathbf{b}=U u_{0} \mathbf{a} u_{0}^{-1}$. If, in addition, $U: \mathcal{M}^{n} \rightarrow \mathcal{M}^{n}$ is invertible then the entries of $u_{0} \mathbf{a} u_{0}^{-1}$ commute with those of $U$ and those of $\mathbf{b}$. It therefore remains to compare ascent or descent of $\mathbf{a}, u_{0} \mathbf{a} u_{0}^{-1}$ and $\mathbf{b}=U u_{0} \mathbf{a} u_{0}^{-1}$.

Corollary 7.5. Let $A$ and $B$ be commutative $R$-subalgebras of $\operatorname{End}_{\mathcal{A}}(\mathcal{M})$ containing the $n$-tuples $\mathbf{a}$ and $\mathbf{b}$, respectively, such that $\mathbf{b} u_{0}=U u_{0} \mathbf{a}$ for some $U \in \mathrm{M}_{n}(B)$ and some $u_{0} \in \operatorname{Aut}_{\mathcal{A}}(\mathcal{M})$. Then $\left(u_{0}, U\right)$ induces a cochain map $u^{(r)}: \mathcal{M}^{(r)}\left(\partial_{\mathbf{a}}\right) \rightarrow \mathcal{M}^{(r)}\left(\partial_{\mathbf{b}}\right)$ for each $r \geq 1$. Moreover, if $U \in \mathrm{GL}_{n}(B)$ then $u^{(r)}$ is an isomorphism for $r \geq 1$ so that $\mathbf{a}$ and $\mathbf{b}$ have the same ascent and the same descent.

Proof. Since $U \in \mathrm{M}_{n}(B)$, the conditions of Proposition 7.3 are obviously satisfied, and the assertion for $r=1$ follows. Moreover, since the entries of $U$ commute and they also commute with the entries of $\mathbf{b}$ it follows that

$$
\begin{aligned}
b_{i} u\left(v e_{J}\right) & =\sum_{I} b_{i} u_{I J} u_{0}(v) f_{I}=\sum_{I} u_{I J} b_{i} u_{0}(v) f_{I} \\
& =\sum_{I, j} u_{I J} u_{i j} u_{0}\left(a_{j} v\right) f_{I} \\
& =\sum_{j, I} u_{i j} u_{I J} u_{0}\left(a_{j} v\right) f_{I}=\sum_{j} u_{i j} u\left(a_{j} v e_{J}\right),
\end{aligned}
$$

which means that $b_{i} u=\sum_{j} u_{i j} u a_{j}$.

Now proceed by induction on $r$. Suppose that $u^{(r)}: \mathcal{M}^{(r)}(\mathbf{a}) \rightarrow \mathcal{M}^{(r)}(\mathbf{b})$ has already been defined such that $\partial_{\mathbf{b}} u^{(r)}=u^{(r)} \partial_{\mathbf{a}}$ and that $b_{i} u^{(r)}=$ $\sum_{j} u_{i j} u^{(r)} a_{j}$. Then define $u^{(r+1)}: \mathcal{M}^{(r+1)}(\mathbf{a}) \rightarrow \mathcal{M}^{(r+1)}(\mathbf{b})$ by $u^{(r+1)}(x \otimes$ $\left.e_{I}\right)=\sum_{J} u_{J I} u^{(r)} x \otimes f_{J}$. The commutativity conditions and the induction hypothesis then imply that

$$
\begin{aligned}
\partial_{\mathbf{b}}^{(r+1)} u^{(r+1)}\left(x \otimes e_{I}\right) & =\sum_{j, J} b_{j} \partial_{\mathbf{b}}^{(r)} u_{J I} u^{(r)} x \otimes\left(f_{j} \wedge f_{J}\right) \\
& =\sum_{j, J} b_{j} u_{J I} u^{(r)} \partial_{\mathbf{a}}^{(r)} x \otimes\left(f_{j} \wedge f_{J}\right) \\
& =\sum_{i, j, J} u_{j i} u_{J I} u^{(r)} a_{i} \partial_{\mathbf{a}}^{(r)} x \otimes\left(f_{j} \wedge f_{J}\right) \\
& =u^{(r+1)}\left(\sum_{i} a_{i} \partial_{\mathbf{a}}^{(r)} x \otimes\left(e_{i} \wedge e_{I}\right)\right)
\end{aligned}
$$




$$
=u^{(r+1)} \partial_{\mathbf{a}}^{(r+1)}\left(x \otimes e_{I}\right)
$$

and also

$$
\begin{aligned}
b_{i} u^{(r+1)}\left(x \otimes e_{I}\right) & =\sum_{J} u_{J I} b_{i} u^{(r)} x \otimes f_{J} \\
& =\sum_{J, i} u_{J I} u_{i j} u^{(r)}\left(a_{j} x\right) \otimes f_{J}=\sum_{j} u_{i j} u^{(r+1)}\left(a_{j} x \otimes e_{I}\right) .
\end{aligned}
$$

This means that $\partial_{\mathbf{b}}^{(r+1)} u^{(r+1)}=u^{(r+1)} \partial_{\mathbf{a}}^{(r+1)}$ and $b_{i} u^{(r+1)}=\sum_{j} u_{i j} u^{(r+1)} a_{j}$. If $u_{0} \in \operatorname{Aut}_{\mathcal{A}}(\mathcal{M})$ and $U \in \mathrm{GL}_{n}(B)$ then one shows directly that the inverse of $u^{(r)}$ is induced as above by $\left(u_{0}^{-1},\left[u_{0}^{-1} u_{i j} u_{0}\right]^{-1}\right)$ for each $r \geq 1$. There is another, more conceptual way to show that $u^{(r)}$ is an isomorphism. It is easy to see that $\left(u_{0}, i d\right)$ induces an isomorphism $\mathcal{M}^{(r)}\left(\partial_{\mathbf{a}}\right) \cong \mathcal{M}^{(r)}\left(\partial_{u_{0} \mathbf{a} u_{0}^{-1}}\right)$. The fact that $(i d, U)$ induces an isomorphism $\mathcal{M}^{(r)}\left(\partial_{u_{0} \mathbf{a} u_{0}^{-1}}\right) \cong \mathcal{M}^{(r)}\left(\partial_{\mathbf{b}}\right)$ uses the assumption that the entries of $u_{0} \mathbf{a} u_{0}^{-1}$ commute with those of $U$ and those of $\mathbf{b}$ so that we may assume that all these entries are contained in $B$. The rest is then a consequence of the following general observation. If $\phi: X \rightarrow X^{\prime}$ and $\psi: Y \rightarrow Y^{\prime}$ are maps of differential graded $B$-modules then $\phi \otimes_{B} \psi: X \otimes_{B} Y \rightarrow X^{\prime} \otimes_{B} Y^{\prime}$ is a map of bicomplexes and $D\left(\phi \otimes_{B} \psi\right)$ : $D\left(X \otimes_{B} Y\right) \rightarrow\left(X^{\prime} \otimes_{B} Y^{\prime}\right)$ is a homomorphism of the associated diagonal complexes. Moreover, if $\phi$ and $\psi$ are isomorphisms then so are $\phi \otimes_{B} \psi$ and $D\left(\phi \otimes_{B} \psi\right)$.

\section{Invariance Properties.}

8.1. In case $u_{0}=i d$, the assumption that $\mathbf{b}=U \mathbf{a}$ with $U \in \mathrm{GL}_{n}(A)$ in 7.5 clearly implies that $\mathbf{a}$ and $\mathbf{b}$ generate the same ideal in $A$. The question now arises whether an $n$-tuple a and an $m$-tuple $\mathbf{b}$, which generate the same ideal in $A$, also have the same ascent and the same descent. This is indeed the case, as we shall demonstrate using localization arguments together with the following basic result.

Theorem. Let $A$ be a commutative $R$-subalgebra of $\operatorname{End}_{\mathcal{A}}(\mathcal{M})$ containing the $n$-tuple $\mathbf{a}$ and the $m$-tuple $\mathbf{b}$. Suppose $\mathbf{a}$ and $\mathbf{b}$ generate the same ideal in $A$ and that $\mathbf{b}=U \mathbf{a}^{\prime}$ for some $\mathbf{a}^{\prime} \subseteq \mathbf{a}$ and some $U \in \mathrm{GL}_{m}(A)$. Then $K\left(\mathcal{M}, \partial_{\mathbf{a}}\right) \cong K\left(\mathcal{M}, \partial_{\mathbf{b}}\right) \otimes K\left(R^{n-m}, 0\right)$, hence $H\left(K\left(\mathcal{M}, \partial_{\mathbf{a}}\right)\right) \cong H\left(K\left(\mathcal{M}, \partial_{\mathbf{b}}\right)\right)$ $\otimes \Lambda\left(R^{n-m}\right)$. Moreover, $\mathbf{a}$ and $\mathbf{b}$ have the same ascent and the same descent.

Proof. In view of Corollary 7.5 we may assume that $\mathbf{b}=\left(a_{1}, a_{2}, \ldots, a_{m}\right)$, and that $\mathbf{a}$ and $\mathbf{b}$ generate the same ideal in $A$. Thus, if the $n$-tuple $\mathbf{c}=\mathbf{b} \cup \mathbf{0}$ is obtained from $\mathbf{b}$ by adjoining $n-m$ zeros, then there is a system of equations $\sum_{j=1}^{i} u_{i j}(\mathbf{a}) a_{j}=c_{i}$ with $u_{i i}=1$ for $i=1,2, \ldots, n$. The matrix 
$U=\left[u_{i j}\right]$ is in $\mathrm{GL}_{n}(A)$, since $\operatorname{det} U=1$. So, by Corollary 7.5 we have

$$
K\left(\mathcal{M}, \partial_{\mathbf{a}}\right) \cong K\left(\mathcal{M}, \partial_{\mathbf{c}}\right) \cong K\left(\mathcal{M}, \partial_{\mathbf{b}}\right) \otimes K\left(R^{n-m}, 0\right)
$$

as complexes and thus $H\left(K\left(\mathcal{M}, \partial_{\mathbf{a}}\right)\right) \cong H\left(K\left(\mathcal{M}, \partial_{\mathbf{b}}\right)\right) \otimes \Lambda\left(R^{n-m}\right)$ as graded modules. Moreover, a and $\mathbf{c}$ have the same ascent and the same descent, and it then remains to show that the same is true for $\mathbf{c}$ and $\mathbf{b}$. By induction we may assume that $\mathbf{c}$ is obtained from $\mathbf{b}$ by adjoining a single zero. For this purpose let $D(\mathbf{b})=K\left(A, \partial_{\mathbf{b}}\right)$ and $E=K(R, 0)$, so that $D(\mathbf{c})=K\left(A, \partial_{\mathbf{c}}\right)=$ $D(\mathbf{b}) \otimes E$ and moreover $K\left(\mathcal{M}, \partial_{\mathbf{b}}\right)=\mathcal{M} \otimes_{A} D(\mathbf{b}), K\left(\mathcal{M}, \partial_{\mathbf{c}}\right)=\mathcal{M} \otimes_{A} D(\mathbf{c}) \cong$ $\mathcal{M} \otimes_{A} D(\mathbf{b}) \otimes E$.

By the results of Section 4, we only have to analyse the diagram

$$
\begin{aligned}
& \mathcal{M}_{m}^{(r)}(\mathbf{c}) \\
& \partial_{m}^{(r)}(\mathbf{c}) \downarrow \\
& \mathcal{M}_{m+1}^{(r)}(\mathbf{c}) \stackrel{\partial_{0}^{r+1}(\mathbf{c})}{\longrightarrow} \mathcal{M}_{m+1}^{r}(\underline{c}) \otimes_{A} D_{1}(\mathbf{c})
\end{aligned}
$$

to solve the ascent problem. First notice that $D(\mathbf{c})=D(\mathbf{b}) \otimes E$ means in particular that

$$
D_{i}(\mathbf{c})=\left(D_{i}(\mathbf{b}) \otimes E_{0}\right) \oplus\left(D_{i-1}(\mathbf{b}) \otimes E_{1}\right) \cong D_{i}(\mathbf{b}) \oplus D_{i-1}(\mathbf{b})
$$

for $0 \leq i \leq m+1$, where of course $D_{j}(\mathbf{b})=0$ for $j<0$ and for $j>m$. Moreover, since the differential of $E$ is zero, the diagram

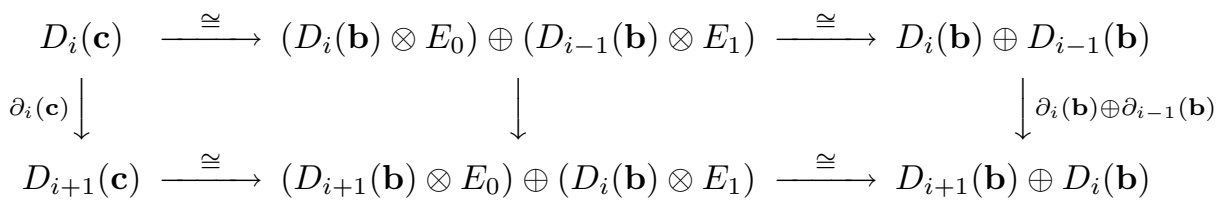

commutes for every $i, 0 \leq i \leq m$. More generally, since $D_{i}^{(r)}(\mathbf{c}) \cong$ $\otimes_{A}^{r}\left(D_{i}(\mathbf{b}) \oplus D_{i-1}(\mathbf{b})\right)$, the $r$-fold tensor product, we see that $\partial_{i}^{(r)}(\mathbf{c})=$ $\otimes_{A}^{r}\left(\partial_{i}(\mathbf{b}) \oplus \partial_{i-1}(\mathbf{b})\right)$. In particular for $i=m$ and for $i=0$, after expressing the right hand parts as direct sums, the diagrams

$$
\begin{aligned}
& D_{m}^{(r)}(\mathbf{c}) \stackrel{\cong}{\longrightarrow} Z \oplus D_{m-1}^{(r)}(\mathbf{b}) \quad, \quad D_{0}^{(r)}(\mathbf{c}) \stackrel{\cong}{\longrightarrow} D_{0}^{(r)}(\mathbf{b}) \\
& \partial_{m}^{(r)}(\mathbf{c}) \downarrow \quad \downarrow\left(0, \partial_{m-1}^{(r)}(\mathbf{b})\right) \quad \partial_{0}^{(r)}(\mathbf{c}) \downarrow \quad \downarrow\left(\partial_{0}^{(r)}(\mathbf{b}), 0\right)^{t} \\
& D_{m+1}^{(r)}(\mathbf{c}) \stackrel{\cong}{\longrightarrow} D_{m}^{(r)}(\mathbf{b}) \quad D_{1}^{(r)}(\mathbf{c}) \stackrel{\cong}{\longrightarrow} D_{1}^{(r)}(\mathbf{b}) \oplus U
\end{aligned}
$$


commute. It follows that the differential $\partial_{m}^{(r)}(\mathbf{c})$ is related to $\partial_{m-1}^{(r)}(\mathbf{b})$ by an isomorphism of the form

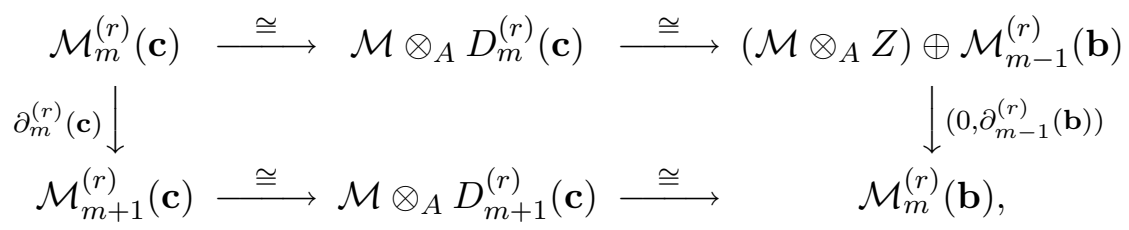

while the relation between the differentials $\partial_{0}^{r+1}(\mathbf{c})$ and $\partial_{0}^{r+1}(\mathbf{b})$ is given by

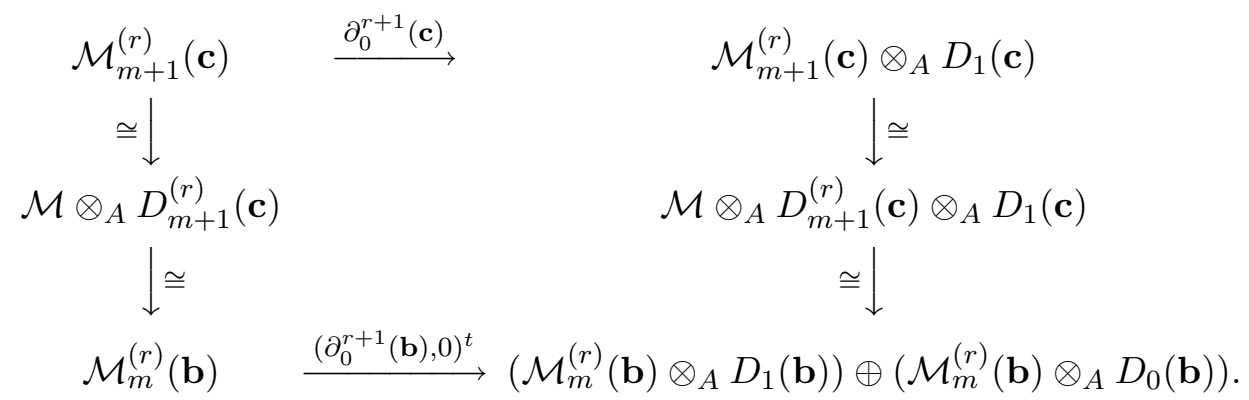

With the proper identifications we therefore get the matrix equation

$$
\partial_{0}^{r+1}(\mathbf{c}) \partial_{m}^{(r)}(\mathbf{c})=\left(\begin{array}{c}
\partial_{0}^{r+1}(\mathbf{b}) \\
0
\end{array}\right)\left(\begin{array}{ll}
0 & \partial_{m-1}^{(r)}(\mathbf{b})
\end{array}\right)=\left(\begin{array}{cc}
0 & \partial_{0}^{r+1}(\mathbf{b}) \partial_{m-1}^{(r)}(\mathbf{b}) \\
0 & 0
\end{array}\right)
$$

This shows that $\operatorname{ker} \partial_{m}^{(r)}(\mathbf{c})=\left(\mathcal{M} \otimes_{A} Z\right) \oplus \operatorname{ker} \partial_{m}^{(r)}(\mathbf{b})$ and $\operatorname{ker}\left(\partial_{0}^{r+1}(\mathbf{c}) \partial_{m}^{(r)}(\mathbf{c})\right)$ $=\left(\mathcal{M} \otimes_{A} Z\right) \oplus \operatorname{ker}\left(\partial_{0}^{r+1}(\mathbf{b}) \partial_{m}^{(r)}(\mathbf{b})\right)$, and that the inclusion of the former into the latter is componentwise. Hence, $\operatorname{ker} \partial_{m}^{(r)}(\mathbf{c})=\operatorname{ker}\left(\partial_{0}^{r+1}(\mathbf{c}) \partial_{m}^{(r)}(\mathbf{c})\right)$ if and only if $\operatorname{ker} \partial_{m}^{(r)}(\mathbf{b})=\operatorname{ker}\left(\partial_{0}^{r+1}(\mathbf{b}) \partial_{m}^{(r)}(\mathbf{b})\right)$, which implies the invariance of ascent.

To solve the descent problem we have, again by the results of Section 4, to analyse the diagram

$$
\begin{aligned}
\mathcal{M}_{0}^{(r)}(\mathbf{c}) \otimes_{A} D_{m}(\mathbf{c}) \stackrel{\partial_{m}^{r+1}(\mathbf{c})}{\longrightarrow} & \mathcal{M}_{0}^{(r)}(\mathbf{c}) \otimes_{A} D_{m+1}(\mathbf{c}) \\
& \downarrow_{0}^{(r)}(\mathbf{c}) \\
& \mathcal{M}_{1}^{(r)}(\mathbf{c}) \otimes_{A} D_{m+1}(\mathbf{c}) .
\end{aligned}
$$


Now, $\partial_{m}^{r+1}(\mathbf{c})$ is related to $\partial_{m-1}^{r+1}(\mathbf{b})$ by the isomorphism
$\mathcal{M}_{0}^{(r)}(\mathbf{c}) \otimes_{A} D_{m}(\mathbf{c})$
$\stackrel{\partial_{m}^{r+1}(\mathbf{c})}{\longrightarrow}$
$\mathcal{M}_{0}^{(r)}(\mathbf{c}) \otimes_{A} D_{m+1}(\mathbf{c})$
$\cong \downarrow$

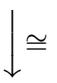
$\mathcal{M} \otimes_{A} D_{0}^{(r)}(\mathbf{c}) \otimes_{A} D_{m}(\mathbf{c})$
$\mathcal{M} \otimes_{A} D^{(r)}(\mathbf{c}) \otimes_{A} D_{m+1}(\mathbf{c})$
$\cong \downarrow$
$\downarrow \cong$
$\left(\mathcal{M}_{0}^{(r)}(\mathbf{b}) \otimes_{A} D_{m-1}(\mathbf{b})\right) \oplus V \stackrel{\left(\partial_{m-1}^{r+1}(\mathbf{b}), 0\right)}{\longrightarrow} \quad \mathcal{M}_{0}^{(r)}(\mathbf{b}) \otimes_{A} D_{m}(\mathbf{b})$

with $V=\mathcal{M}^{(r)}(\mathbf{b}) \otimes_{A} D_{m}(\mathbf{b})$, while $\partial_{0}^{(r)}(\mathbf{c})$ and $\partial_{0}^{(r)}(\mathbf{b})$ are related by

$$
\begin{array}{rlrl}
\mathcal{M}_{0}^{(r)}(\mathbf{c}) & \otimes_{A} D_{m+1}(\mathbf{c}) & \stackrel{\partial_{0}^{(r)}(\mathbf{c})}{\longrightarrow} & \mathcal{M}_{1}^{(r)}(\mathbf{c}) \otimes_{A} D_{m+1}(\mathbf{c}) \\
\cong \downarrow & & \downarrow \cong \\
\mathcal{M} \otimes_{A} D_{0}^{(r)}(\mathbf{c}) \otimes_{A} D_{m+1}(\mathbf{c}) & & \mathcal{M} \otimes_{A} D_{1}^{(r)}(\mathbf{c}) \otimes_{A} D_{m+1}(\mathbf{c}) \\
\cong \downarrow & \stackrel{\left(\partial_{0}^{(r)}(\mathbf{b}), 0\right)^{t}}{\longrightarrow} & \left(\mathcal{M}_{1}^{(r)}(\mathbf{b}) \otimes_{A} D_{m}(\mathbf{b})\right) \oplus W \\
\mathcal{M}_{0}^{(r)}(\mathbf{b}) \otimes_{A} D_{m}(\mathbf{b}) &
\end{array}
$$

if $W=\mathcal{M} \otimes_{A} U \otimes_{A} D_{m}(\mathbf{b})$. Considering the isomorphisms as identifications we have

$$
\partial_{0}^{(r)}(\mathbf{c}) \partial_{m}^{r+1}(\mathbf{c})=\left(\begin{array}{cc}
\partial_{0}^{(r)}(\mathbf{b}) \\
0
\end{array}\right)\left(\begin{array}{ll}
\partial_{m-1}^{r+1}(\mathbf{b}) & 0
\end{array}\right)=\left(\begin{array}{cc}
\partial_{0}^{(r)}(\mathbf{b}) \partial_{m-1}^{r+1}(\mathbf{b}) & 0 \\
0 & 0
\end{array}\right) .
$$

Thus, $\operatorname{im} \partial_{0}^{(r)}(\mathbf{c})=\operatorname{im} \partial_{0}^{(r)}(\mathbf{b})$ and $\operatorname{im}\left(\partial_{0}^{(r)}(\mathbf{c}) \partial_{m}^{r+1}(\mathbf{c})\right)=\operatorname{im}\left(\partial_{0}^{(r)}(\mathbf{b}) \partial_{m}^{r+1}(\mathbf{b})\right)$. In particular, we may conclude now that $\operatorname{im}\left(\partial_{0}^{(r)}(\mathbf{c}) \partial_{m}^{r+1}(\mathbf{c})\right)=\operatorname{im} \partial_{0}^{(r)}(\mathbf{c})$ if and only if $\operatorname{im}\left(\partial_{0}^{(r)}(\mathbf{b}) \partial_{m-1}^{r+1}(\mathbf{b})\right)=\operatorname{im} \partial_{0}^{(r)}(\mathbf{b})$, establishing the invariance of descent.

Corollary 8.2. Let $R$ be a field and let $L$ be a finite dimensional subspace of commuting elements in $\operatorname{End}_{\mathcal{A}}(\mathcal{M})$. Any two finite spanning subsets of $L$ have the same ascent and the same descent.

Proof. If $\mathbf{a}$ and $\mathbf{b}$ are two finite spanning subsets of $L$ then they generate the same commutative subalgebra $A$ and the same ideal in it. Moreover, they each contain a basis $\mathbf{a}^{\prime}$ and $\mathbf{b}^{\prime}$ of $L$, so that $\mathbf{b}^{\prime}=U \mathbf{a}^{\prime}$ for some $U \in \mathrm{GL}_{n}(R)$, where $n=\operatorname{dim}_{R} L$. The assertion now follows from Theorem 8.1.

8.3. Additional conditions on the commuting $n$-tuple a or the commutative subalgebra $A$ of $\operatorname{End}_{\mathcal{A}}(\mathcal{M})$ it generates may give tighter invariance results. Such a situation occurs when $A$ is a commutative local algebra; this happens for example when $R$ is a field and a consists of nilpotent endomorphisms. 
Theorem. Suppose $A$ is a commutative local subalgebra of $\operatorname{End}_{\mathcal{A}}(\mathcal{M})$ with maximal ideal $M$ and residue class field $F$ and let $I$ be a finitely generated ideal of $A$. Then any two finite generating subsets of I have the same ascent and the same descent.

Proof. The $n$-tuple $\mathbf{a}=\left(a_{1}, a_{2}, \ldots, a_{n}\right) \subset I$ forms a generating subset for the ideal $I$ of the commutative local algebra $A$ if and only if the residue classes $\tilde{\mathbf{a}}=\left(\tilde{a}_{1}, \tilde{a}_{2}, \ldots, \tilde{a}_{n}\right)$ span $I / M I$ as a vector space over the field $F=A / M$, i.e. if and only if $\tilde{\mathbf{a}}$ contains an $F$-basis of $I / M I$. This follows from Nakayama's Lemma $[\mathbf{A}, \mathbf{S}]$. Thus, if this is the case, then a contains a subset $\mathbf{a}^{\prime}$ which generates the ideal $I$ and such that $\tilde{\mathbf{a}}^{\prime}$ is an $F$-basis of $I / M I$. By Theorem 8.1 we see that $\mathbf{a}$ and $\mathbf{b}=\mathbf{a}^{\prime}$ have the same ascent and the same descent. It remains to show that the same is true for $m$-tuples a and $\mathbf{b}$ for which $\tilde{\mathbf{a}}$ and $\tilde{\mathbf{b}}$ are $F$-bases of $I / M I$. But in this case $\mathbf{b}=U \mathbf{a}$ for some matrix $U \in \mathrm{M}_{m}(A)$ with $\tilde{U} \in \mathrm{GL}_{m}(F)$. Then $\widetilde{\operatorname{det} U}=\operatorname{det} \tilde{U} \neq 0$, so that $\operatorname{det} U \notin M$. Hence $\operatorname{det} U$ is invertible and $U \in \mathrm{GL}_{m}(A)$. Again by Theorem 8.1 we conclude that $\mathbf{a}$ and $\mathbf{b}$ have the same ascent and the same descent.

8.4. We are now in a position to prove a general invariance result for any finitely generated ideal in a commutative subalgebra $A$ of $\operatorname{End}_{\mathcal{A}}(\mathcal{M})$.

Theorem. Let $A$ be a commutative $R$-subalgebra of $\operatorname{End}_{\mathcal{A}}(\mathcal{M})$ and let $J$ be a finitely generated ideal of $A$. Then any two finite generating subsets of $J$ have the same ascent and the same descent.

Proof. Suppose that $J$ can be generated by the $n$-tuple $\mathbf{a}=\left(a_{1}, a_{2}, \ldots, a_{n}\right)$ as an ideal of $A$. For any prime ideal $P$ of $A$ let $A_{P}$ and $\mathcal{M}_{P}=A_{P} \otimes_{A} \mathcal{M}$ denote the localizations of $A$ and $\mathcal{M}$ at $P$. The ideal $I=J A_{P}$ of the local ring $A_{P}$ is then generated by a as well. We shall see that

$$
d^{j}(\mathbf{a}, \mathcal{M})=\sup _{P}\left\{d^{j}\left(\mathbf{a}, \mathcal{M}_{P}\right)\right\}
$$

for $j=0,1$. Since the localization functor ()$_{P}: \operatorname{Mod}_{A} \rightarrow \operatorname{Mod}_{A_{P}}$ is exact [A, Proposition 3.9], [S], we see that $K^{(r)}\left(\mathcal{M}, \partial_{\mathbf{a}}\right)_{P} \cong K^{(r)}\left(\mathcal{M}_{P}, \partial_{\mathbf{a}}\right)$, $\mathcal{H}^{(r)}\left(\mathcal{M}, \partial_{\mathbf{a}}\right)_{P} \cong \mathcal{H}^{(r)}\left(\mathcal{M}_{P}, \partial_{\mathbf{a}}\right), \quad \mathcal{L}^{(r)}\left(\mathcal{M}, \partial_{\mathbf{a}}\right)_{P} \cong \mathcal{L}^{(r)}\left(\mathcal{M}_{P}, \partial_{\mathbf{a}}\right)$ and $\mathcal{R}^{(r)}\left(\mathcal{M}, \partial_{\mathbf{a}}\right)_{P} \cong \mathcal{R}^{(r)}\left(\mathcal{M}_{P}, \partial_{\mathbf{a}}\right)$ for all $r \geq 1$. Thus, by Propostions 4.1 and 4.2 , we see that $d^{j}(\mathbf{a}, \mathcal{M}) \geq d^{j}\left(\mathbf{a}, \mathcal{M}_{P}\right)$ for every prime ideal $P$ of $A$. But, by [A, Proposition 3.9], [S], a homomorphism of $A$-modules $f: X \rightarrow Y$ is an isomorphism if and only if for each prime ideal $P$ of $A$ the induced homomorphism of $A_{P}$-modules $f_{P}: X_{P} \rightarrow Y_{P}$ is an isomorphism. By the definition of ascent and descent in 4.3 we conclude that

$$
d^{j}(\mathbf{a}, \mathcal{M})=d^{j}\left(\mathbf{a}, \mathcal{M}_{P}\right)
$$


for some prime ideal $P$ of $A$. If $\mathbf{b}$ is another generating subset of the ideal $J$ then invoke Theorem 8.3 to see that

$$
d^{j}(\mathbf{a}, \mathcal{M})=\sup _{P}\left\{d^{j}\left(\mathbf{a}, \mathcal{M}_{P}\right)\right\}=\sup _{P}\left\{d^{j}\left(\mathbf{b}, \mathcal{M}_{P}\right)\right\}=d^{j}(\mathbf{b}, \mathcal{M})
$$

which proves our assertion.

\section{References}

[A] M. Atiyah and I.G. Macdonald, Introduction to Commutative Algebra, Benjamin, New York, 1972.

[B] D. Benson, Representations and Cohomology, Cambridge University Press, Cambridge, 1991.

[BT] T. Bhattacharyya, On tuples of commuting compact operators, Publ. RIMS. Kyoto Univ., 32 (1996), 785-795.

[BH] W. Bruns and J. Herzog, Cohen-Macaulay Rings, Cambridge University Press, Cambridge, 1993.

[C] R.B. Curto, Spectral Theory of Elementary Operators, in 'Elementary operators and applications', ed. M. Mathieu, Proceedings of International Workshop, World Scientific, Singapore, (1992), 3-52.

[P] R.S. Pierce, Associative Algebras, Springer, New York, 1982.

[R] B.P. Rynne, Multiparameter spectral theory and Taylor's joint spectrum in Hilbert space, Proc. Edin. Math. Soc., 31 (1988), 122-144.

[S] J.P. Serre, Algèbre Locale et Multiplicités, Springer Lecture Notes, 11 (1965).

[TAE] A.E. Taylor, Introduction to Functional Analysis, Wiley and Sons, New York, 1958.

[T] J.L. Taylor, A joint spectrum for several commuting operators, J. Funct. Anal., 6 (1970), 172-191.

Received February 20, 1998 and revised August 18, 1998. This research supported in part by NSERC of Canada and the Ministry of Science and Technology of Slovenia.

Department of Mathematics, Statistics and Computing Science

DALHOUSIE UNIVERSITY

HALIFAX, N.S. B3H 3J5

CANADA

E-mail address: luzius@mscs.dal.ca

Department of Mathematics

UNIVERSITY OF LJUBLJANA

61000 LJUBLJANA

Slovenia 